\title{
A recombinant human IgG1 Fc multimer designed to mimic the active fraction of IVIG in autoimmunity
}

\author{
Xiaoyu Zhang, ${ }^{1}$ Jane Owens, ${ }^{2}$ Henrik S. Olsen, ${ }^{3}$ Edward So, ${ }^{1}$ Erin Burch, ${ }^{1}$ Mark C. McCroskey, ${ }^{4}$ \\ Xianfeng Li, ${ }^{2}$ Gregory L. Weber, ${ }^{2}$ Donald Bennett, ${ }^{2}$ Denis Rybin, ${ }^{2}$ Hua Zhou, ${ }^{1}$ Haiping Hao, ${ }^{5}$ \\ Emmanuel Y. Mérigeon, ${ }^{3}$ David S. Block, ${ }^{4}$ Gregory LaRosa, ${ }^{2}$ and Scott E. Strome ${ }^{1}$ \\ 'Department of Otorhinolaryngology-Head and Neck Surgery, University of Maryland School of Medicine, Baltimore, \\ Maryland, USA. ${ }^{2}$ ffizer Inc., Cambridge, Massachusetts, USA. ${ }^{3}$ Cliknik Inc., Baltimore Maryland, USA. ${ }^{4}$ Pfizer Inc., Groton, \\ Connecticut, USA. 5Johns Hopkins School of Medicine, Baltimore, Maryland, USA.
}

The antiinflammatory effects of i.v. Ig (IVIC) in the treatment of autoimmune disease are due, in part, to the Fc fragments of Ig aggregates. In order to capitalize on the known antiinflammatory and tolerogenic properties of Ig Fc aggregates, we created a recombinant human IgG1 Fc multimer, GL-2045. In vitro, GL-2045 demonstrated high-avidity binding to Fc receptors, blocked the binding of circulating immune complexes from patients with rheumatoid arthritis to human Fc $\gamma$ receptors (FcyRs), and inhibited antibody-mediated phagocytosis at log order-lower concentrations than IVIC. In vivo, administration of GL-2045 conferred partial protection against antibody-mediated platelet loss in a murine immune thrombocytopenic purpura (ITP) model. CL-2045 also suppressed disease activity in a therapeutic model of murine collagen-induced arthritis (CIA), which was associated with reduced circulating levels of IL-6. Furthermore, GL2045 administration to nonhuman primates (NHPs) transiently increased systemic levels of the antiinflammatory cytokines IL-10 and IL-1RA, reduced the proinflammatory cytokine IL-8, and decreased surface expression of CD14 and HLA-DR on monocytes. These findings demonstrate the immunomodulatory properties of $\mathrm{CL}-2045$ and suggest that it has potential as a treatment for autoimmune and inflammatory diseases, as a recombinant alternative to IVIC.

Conflict of interest: SES is a cofounder, consultant, and stockholder in Cliknik Inc., a biotechnology company. He receives royalties for intellectual property, related to B7-H1 (PD-L1), licensed by the Mayo Clinic College of Medicine to third parties. He receives research support from Pfizer and Cliknik through sponsored research agreements through the University of Maryland, Baltimore. He also serves as a paid consultant to Astra Zeneca and Roche. HSO, EYM, and DSB are employees of Cliknik. J0, XL, GLR, DB, DR, and GL are employees of Pfizer Inc. MCM has retired from Pfizer.

License: Copyright 2019, American Society for Clinical Investigation.

Submitted: May 7, 2018

Accepted: December 11, 2018

Published: January 24, 2019

\section{Reference information:}

JCI Insight. 2019;4(2):e121905.

https://doi.org/10.1172/jici.

insight.121905.

\section{Introduction}

I.v. Ig (IVIG) is a marketed product composed of Igs from thousands of human donors (1). While the mechanism by which IVIG restores functional humoral immunity in patients with antibody deficiencies are self-evident, the mechanisms by which IVIG regulates tolerance in patients with autoimmune disease are much more speculative (2-18). The practical consequence of such mechanistic diversity and/or uncertainty has been the historic inability to develop a recombinant drug that is capable of recapitulating the antiinflammatory properties of IVIG. Therefore, the medical community is left in a situation where intra- and interproduct variability are uncomfortable realities; where life-threatening toxicities, such as thrombosis and acute hemolysis, are infrequent yet well documented; and where mechanistic studies are complicated by product diversity $(19,20)$. Additionally, while clinical use of IVIG fractions with purported enhanced antiinflammatory activity might prove mechanistically intriguing (e.g., $\alpha-2,6$ sialylated IVIG), the results reported to date are controversial $(16,21-25)$. Furthermore, the additional steps required for purification are technically challenging, will likely only increase the price of an already expensive therapy, and do not address problems of demand or blood borne pathogen infection risks.

The overwhelming clinical need for a product that could mediate the antiinflammatory properties of IVIG prompted us to embark on a drug discovery process that resulted in the development of M-045, a recombinant mouse IgG2a-based fusion protein bearing the human IgG2 hinge domain on its C-terminus, which induces the formation of highly ordered Fc-multimers (26). M-045 avidly binds all murine Fc $\gamma$ receptors (Fc $\gamma \mathrm{Rs}$ ) and mediates protection against platelet loss in a model of immune thrombocytopenic purpura (ITP), and therapeutic efficacy in a murine collagen-induced arthritis (CIA) model. Importantly, different scientific teams recapitulated the validity of these findings in models of myasthenia gravis and experimental autoimmune neuritis $(27,28)$. 
Based on these findings, we developed a human analogue of M-045, called GL-2045, in preparation for anticipated first in human (FIH) studies and recently reported that GL-2045 inhibits complement activation in vitro (29). Here, we show that GL-2045 binds with high avidity to the low-affinity human Fc $\gamma$ Rs and interferes with the interactions of immune complexes (IC) with Fc $\gamma$ Rs. In vivo studies in murine autoimmune disease models demonstrate the efficacy and enhanced potency of GL-2045 compared with IVIG. In healthy rats and nonhuman primates (NHPs), GL-2045, across a broad range of doses, induced immunomodulatory effects, as shown by changes in the concentration of circulating cytokines and chemokines. These data provide a basis for the continued preclinical evaluation of GL-2045 in order to support advancement to clinical studies of human inflammatory and autoimmune diseases.

\section{Results}

GL-2045 is composed of highly ordered $F_{C}$-multimers and binds to $F_{c}$ receptor-expressing immune cells from different species. GL-2045 was designed by linking the human IgG2 hinge region (ERKCCVECPPCP) to the C-terminus of human IgG1 Fc, which contains hinge $\mathrm{CH} 2$ and $\mathrm{CH} 3$ domains (Figure 1A). SDS-PAGE analysis revealed that nondenatured GL-2045 had multiple distinct ladder-like bands. Under reducing conditions, GL-2045 demonstrated a band between 30-40 kDa, consistent with the predicted size of the glycosylated peptide, and a second light band at approximately $60 \mathrm{kDa}$, which most likely represents a nonreduced doublet (Figure 1B). GL-2045 bound to CHO cell clones expressing human Fc $\gamma$ RIIa, Fc $\gamma$ RIIb, and Fc $\gamma$ RIIIa, while G001, a recombinant human Fc homodimer control, demonstrated only limited interactions (30). The ability of GL-2045 to interact with these Fc $\gamma$ Rs was confirmed by binding studies with human immune cells, where it bound to B cells, NK cells, monocytes, and granulocytes but not to T cells that lack Fc $\gamma$ Rs (Figure 1C). Furthermore, GL-2045 also bound to immune cells originating from mice, rats, and NHPs, presumably through FcR engagement, providing justification for preclinical evaluation of GL-2045 in immunocompetent murine disease models and in healthy rats and NHPs (Figure 1C for NHP and mouse immune cell binding and rat immune cell binding in ref. 30). Importantly, different preparations of GL-2045 (manufactured by Gliknik and Pfizer), demonstrated consistent multimerization signatures, as shown by SDS-PAGE and gel filtration analyses, and similar patterns of binding to human immune cells, indicating the consistency of the compound manufacturing process and thereby mitigating concerns regarding batch-specific treatment responses (Supplemental Figure 1; supplemental material available online with this article; https://doi.org/10.1172/jci.insight.121905DS1).

GL-2045 avidly binds the canonical FCYRs, FCRL5, and FcRn but not DC-SIGN. We employed biolayer interferometry (BLI) and surface plasmon resonance (SPR) to better define the temporal interactions of GL-2045 with individual human FcRs. These data demonstrate that GL-2045 has high avidity to all of the human canonical FcyRs evaluated, with apparent binding affinity $\left(\mathrm{K}_{D}\right)$ values in the $1 \times 10^{-11}$ to $1 \times 10^{-12} \mathrm{M}$ range (Table 1 and Supplemental Figure 2). Importantly, these high levels of avidity were almost exclusively attributable to slower rates of dissociation, as shown by comparison of the dissociation profiles in the sensograms for GL-2045 vs. IVIG and human IgG1 and associated dissociation rate (Kd) values, for binding to Fc $\gamma$ RIIIa, as a representative example low affinity Fcy receptor (Supplemental Figure 2, A-C, and Table 1).

Next, we sought to evaluate the interactions of GL-2045 with several of the noncanonical FcRs, such as FcRL5, FcRn, and DC-SIGN (31-33). GL-2045 interactions with FcRL5 were analogous to those of the low-affinity canonical Fc $\gamma$ Rs (Table 1). While GL-2045 bound to FcRn at a $\mathrm{pH}$ of 6 , the $\mathrm{K}_{D}$ values were higher than those obtained for the binding to the canonical Fc $\gamma$ Rs, suggesting that GL-2045 has weaker avidity for FcRn compared with FcyRs. Additionally, because of the potential role of DC-specific intercellular adhesion molecule-3-grabbing nonintegrin (DC-SIGN)engagement by $\alpha-2,6$ sialylated Fc in mediating the antiinflammatory functions of IVIG (8), we evaluated the ability of both IVIG and GL-2045 to engage this molecule. Neither GL-2045 nor IVIG bound to DC-SIGN, while an ICAM3-positive control demonstrated high-affinity binding (Supplemental Figure 2D).

Finally, to demonstrate that GL-2045 binds to murine, rat, and NHP Fc $\gamma$ Rs, in preparation for rodent and NHP in vivo studies, we characterized the binding of GL-2045 to these different species of canonical Fc $\gamma$ Rs and FcRn (Table 1). GL-2045 bound with high avidity to each species of receptors examined, with low rates of dissociation similar to those seen with the human receptors.

GL-2045 blocks $F c-F c \gamma$ interactions, inhibits antibody-dependent phagocytosis and cytotoxicity and actively signals through Fcy RIIa and FcyIIIa. As a first step in evaluating GL-2045 function, we sought to determine its ability to inhibit IC binding to $\mathrm{CHO}$ cells engineered to express the human receptors Fc $\gamma \mathrm{RIIa}, \mathrm{Fc} \gamma \mathrm{RIIb}$, 
A

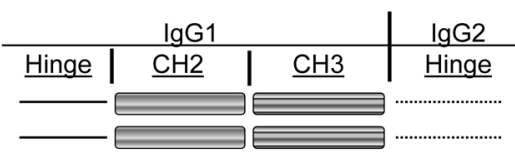

B Non-reduced

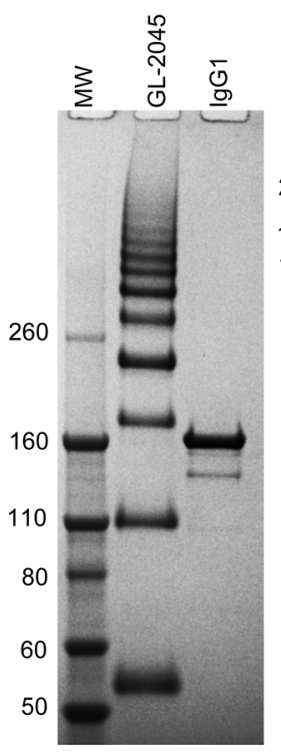

C

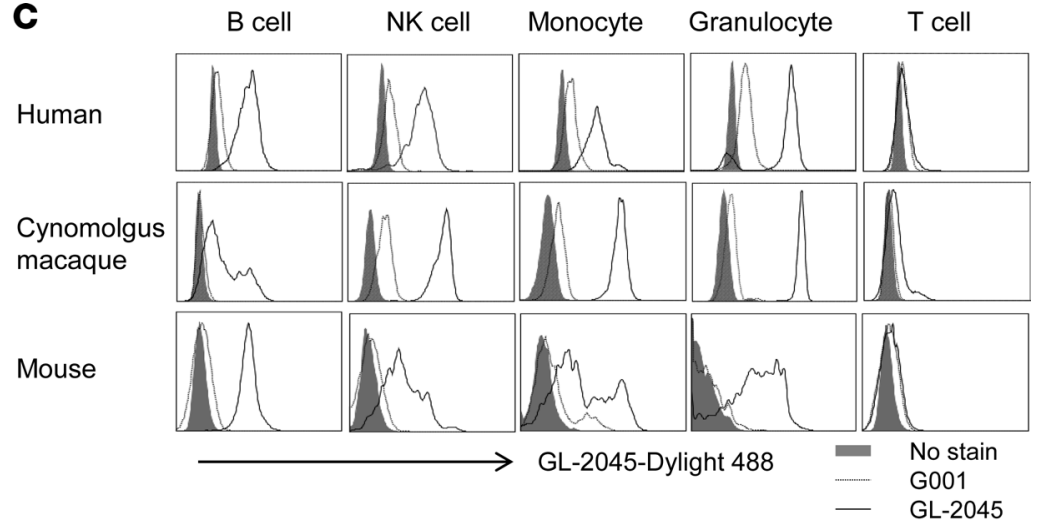

Figure 1. GL-2045 is composed of ordered Fc multimers and effectively binds immune cells from different species. (A) Schematic illustration of the structure of the GL-2045 homodimer composed of the human IgG1 CH2 and CH3 domains and the IgG2 hinge region. (B) SDS-PAGE analysis of GL-2045 under nonreduced and reduced conditions. (C) FACS analysis of GL-2045 and G001 binding to immune cells of human, nonhuman primate (cynomolgus macaque), and mouse origin. For human peripheral blood analysis, T cells/B cells/NK cells were identified as $\operatorname{CD} 3^{+} / \mathrm{CD}^{-} \mathrm{CD}^{-} 9^{+} / \mathrm{CD}^{-} \mathrm{CD} 56^{+}$within the lymphocyte gate, monocytes were characterized as CD14+ cells within the monocyte gate, and granulocytes were identified based on FSC vs. SSC. For cynomolgus macaque peripheral blood, T cells/B cells/NK cells were identified as $C D 3^{+} / \mathrm{CD}^{-} \mathrm{CD}^{-} 9^{+} / \mathrm{CD} 3^{-} \mathrm{CD} 16^{+}$within the lymphocyte gate, monocytes were characterized as $\mathrm{CD} 14^{+}$cells within the monocyte gate, and granulocytes were identified based on FSC vs. SSC. In murine spleen, the markers included

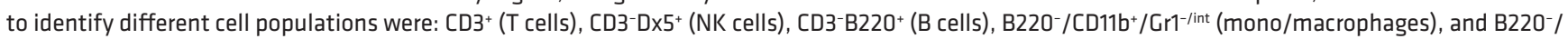
$\mathrm{CD}_{11 \mathrm{~b}^{+}} / \mathrm{Gr} \mathrm{Ch}^{\text {hi }}$ (granulocyte). Data represent 1 of at least 3 different experiments using cells from different donors.

and FcrRIIIa (Figure 2A). CHO-huFc $\gamma$ R cells pretreated with GL-2045 showed significantly diminished ability to bind ICs, found in rheumatoid arthritis (RA) patient serum, compared with those pretreated with the G001 Fc-homodimer control. Furthermore, GL-2045 blocked both antibody-dependent cellular phagocytosis (ADCP) and antibody-dependent cellular cytotoxicity (ADCC) in a dose-dependent manner, with statistically significant effects seen at $10 \mu \mathrm{g} / \mathrm{ml}$ (ADCP) and $100 \mu \mathrm{g} / \mathrm{ml}$ (ADCC), while G001 had no effect (Figure $2 \mathrm{~B})$. IVIG $(10 \mathrm{mg} / \mathrm{ml})$ partially blocked phagocytosis and showed no effect on ADCC, demonstrating the potency of GL-2045 relative to IVIG.

Many of the antiinflammatory effects associated with IVIG are thought to be secondary to active Fc $\gamma R$ signaling, rather than simply passive receptor blockade $(7,12,34)$. To evaluate the ability of GL-2045 to induce Fc $\gamma \mathrm{R}$ signaling, we employed components from ADCP and ADCC reporter bioassays, in which signaling through either Fc $\gamma$ RIIa or Fc $\gamma$ RIIIa on Jurkat cells (T cells) engineered to express Fc $\gamma$ RIIa or Fc $\gamma$ RIIIa, increases luminescence. GL-2045 mediated a bell-shaped dose-response curve in both assays (Figure 2C). We postulate that the diminished responses observed at higher drug concentrations in these reporter bioassays might be due to an overstimulation that leads to signaling pathway desensitization over the time course of the assay. The half-maximal effective concentration $\left(\mathrm{EC}_{50}\right)$ values for GL-2045 were calculated for Fc $\gamma$ RIIa at $0.09 \mathrm{nM}$ and for Fc $\gamma$ RIIIa at $0.9 \mathrm{nM}$. In contrast, while IVIG also mediated a bell-shaped signaling response through Fc $\gamma$ RIIa, its potency and efficacy in both signaling assays was lower than that of GL-2045, as shown by $>3-\log$ order differences in the concentration of IVIG required to induce a weak luminescence signal $\left(\mathrm{EC}_{50} 611 \mathrm{nM}\right.$ and $4200 \mathrm{nM}$ for Fc $\gamma$ RIIa and Fc $\gamma$ RIIIa, respectively). G001 did not mediate signaling at any of the concentrations tested. 
Table 1. Binding of GL-2045 to human, murine, rat, and monkey Fc receptors

\begin{tabular}{|c|c|c|c|}
\hline Human & $\mathbf{K}_{D}^{\mathbf{A}}$ & Ka & Kd \\
\hline Fc $\gamma \mathrm{RI}$ & $<1.00 \times 10^{-12}$ & $1.60 \pm 0.74 \times 10^{6}$ & $<1.00 \times 10^{-7}$ \\
\hline Fc $\gamma R R^{\prime l} a^{B}$ & $<1.00 \times 10^{-12}$ & $1.50 \pm 0.70 \times 10^{6}$ & $<1.00 \times 10^{-7}$ \\
\hline Fc $\gamma R$ IIb & $7.71 \pm 4.51 \times 10^{-11}$ & $1.19 \pm 0.49 \times 10^{6}$ & $7.32 \pm 1.87 \times 10^{-5}$ \\
\hline Fc $\gamma R$ IIIIac $^{c}$ & $4.68 \pm 2.37 \times 10^{-11}$ & $8.82 \pm 3.91 \times 10^{5}$ & $3.66 \pm 2.00 \times 10^{-5}$ \\
\hline FcRn ${ }^{D}$ & $2.44 \pm 2.38 \times 10^{-5}$ & $2.91 \pm 0.31 \times 10^{3}$ & $4.40 \pm 0.12 \times 10^{-2}$ \\
\hline FcRL5 & $1.02 \times 10^{-12}$ & $8.02 \pm 0.09 \times 10^{4}$ & $8.15 \pm 0.02 \times 10^{-8}$ \\
\hline DC-SIGN & NB & & \\
\hline \multicolumn{4}{|l|}{ Murine } \\
\hline Fc $\gamma R$ I & $<1.00 \times 10^{-12}$ & $5.65 \pm 2.57 \times 10^{5}$ & $<1.00 \times 10^{-7}$ \\
\hline Fc $\gamma$ RIIb & $9.57 \pm 8.66 \times 10^{-10}$ & $4.16 \pm 2.84 \times 10^{5}$ & $2.34 \pm 0.73 \times 10^{-4}$ \\
\hline Fc $\gamma R$ RIII & $1.20 \pm 1.12 \times 10^{-9}$ & $6.75 \pm 4.64 \times 10^{5}$ & $4.93 \pm 1.56 \times 10^{-4}$ \\
\hline FcRn & $2.25 \pm 2.55 \times 10^{-9}$ & $4.77 \pm 3.94 \times 10^{5}$ & $4.20 \pm 1.66 \times 10^{-4}$ \\
\hline \multicolumn{4}{|l|}{ Rat } \\
\hline Fc $\gamma \mathrm{RI}$ & $5.22 \pm 3.33 \times 10^{-10}$ & $3.84 \pm 1.78 \times 10^{5}$ & $1.56 \pm 0.59 \times 10^{-4}$ \\
\hline Fc $\gamma$ RIIIa & $5.28 \pm 3.24 \times 10^{-10} 10^{-10}$ & $4.12 \pm 1.72 \times 10^{5}$ & $1.77 \pm 0.68 \times 10^{-4}$ \\
\hline FcRN & $1.26 \pm 2.72 \times 10^{-5}$ & $4.28 \pm 4.23 \times 10^{3}$ & $1.38 \pm 0.74 \times 10^{-3}$ \\
\hline \multicolumn{4}{|l|}{ Monkey } \\
\hline Fc $\gamma$ RIIa & $1.10 \pm 0.48 \times 10^{-10}$ & $8.10 \pm 2.81 \times 10^{5}$ & $7.82 \pm 1.01 \times 10^{-5}$ \\
\hline Fc $\gamma$ RIIb & $9.80 \pm 4.56 \times 10^{-11}$ & $1.18 \pm 0.445 \times 10^{6}$ & $9.86 \pm 1.72 \times 10^{-5}$ \\
\hline Fc $\gamma$ RIIIa & $3.83 \pm 3.56 \times 10^{-11}$ & $9.68 \pm 5.17 \times 10^{5}$ & $2.72 \pm 1.81 \times 10^{-5}$ \\
\hline FcRN & $1.59 \pm 1.31 \times 10^{-55}$ & $3.14 \pm 4.87 \times 10^{4}$ & $1.17 \pm 1.47 \times 10^{-1}$ \\
\hline
\end{tabular}

GL-2045 prevents immune thrombocytopenia and ameliorates CIA in mice. Informed by both our functional studies in vitro and the knowledge that GL-2045 engaged the murine Fc $\gamma$ Rs in a manner similar to their human analogues, we next sought to evaluate the therapeutic potential of GL-2045 in murine models of ITP and CIA. In an ITP model, i.v. administration of GL-2045 at $40 \mathrm{mg} / \mathrm{kg}$ protected mice from anti-CD41mediated platelet loss, as shown by the statistically significant increase in platelet numbers compared with the human albumin control group (Figure 3A). GL-2045 administered at $80 \mathrm{mg} / \mathrm{kg}$ i.v. showed a trend toward protecting against platelet loss, but the effect did not reach statistical significance. Furthermore, consistent with our published data (26), the murine GL-2045 analogue, M-045, administered at $40 \mathrm{mg} / \mathrm{kg}$ i.v., also mediated protection from platelet loss. I.p. administration of $80 \mathrm{mg} / \mathrm{kg}$ of GL-2045 had no effect on platelet numbers, suggesting potential differences in the pharmacokinetic profile caused by different routes of administration. As previously reported (26), i.p. administration of IVIG at $2 \mathrm{~g} / \mathrm{kg}$ was also effective, as shown by the significant increase in platelet numbers compared with the human albumin control group (Figure 3A).

Because the murine ITP model relies on passive antibody administration to mediate platelet loss and the positive effects of GL-2045 were demonstrated in the prevention regimen only, we sought to understand the therapeutic potential of GL-2045 in CIA, where disease is actively induced by collagen vaccination. Treatment with GL-2045, M-045, or IVIG was initiated after onset of arthritis. Analysis of arthritis in all paws revealed that GL-2045 — administered i.v. every 4 days (Q4D) at doses of 10, 30, 50, or $80 \mathrm{mg} / \mathrm{kg}$ — mediated dose-dependent therapeutic effects (Figure 3B). M-045 mediated almost complete disease suppression, which was equivalent to that of dexamethasone. IVIG, given at either $80 \mathrm{mg} / \mathrm{kg}$ i.v. or $2 \mathrm{~g} / \mathrm{kg}$ i.p., did not provide any clinical benefit within the treatment period of this study. While dexamethasone, GL-2045, and M-045 mediated significant effects on the arthritis score, the dexamethasone-treated mice lost weight during treatment. In contrast, GL-2045- and M-045-treated mice gained weight over the same time interval (Figure 3C).

In order to evaluate the influence of GL-2045 on the different stages of active disease, we analyzed the impact of treatment on the paws of animals that were inflamed at the start of therapy (inflamed paws), vs. those that were not swollen but maintained the potential to develop disease (noninflamed paws) (Figure 3 , D and E). GL-2045, M-045, and dexamethasone suppressed the development of disease in the noninflamed 
A

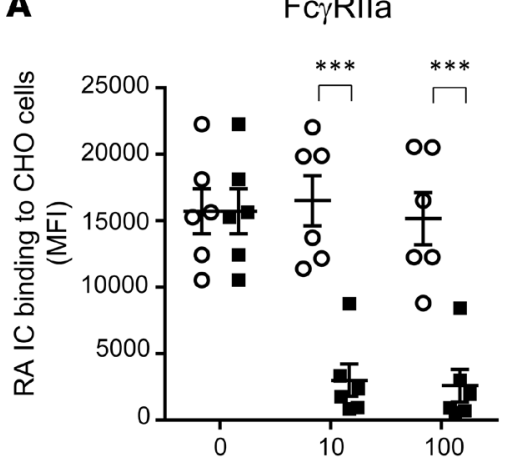

FcyRllb

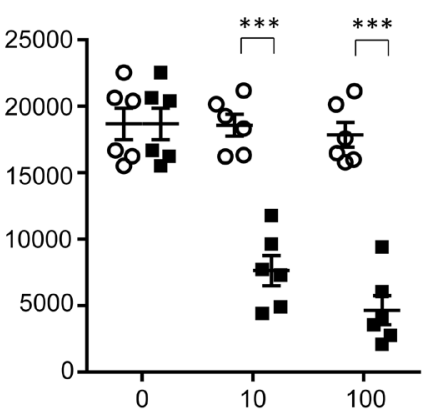

FcyRllla

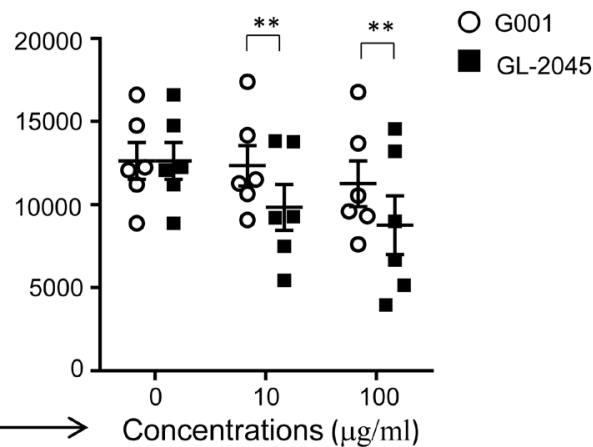

B
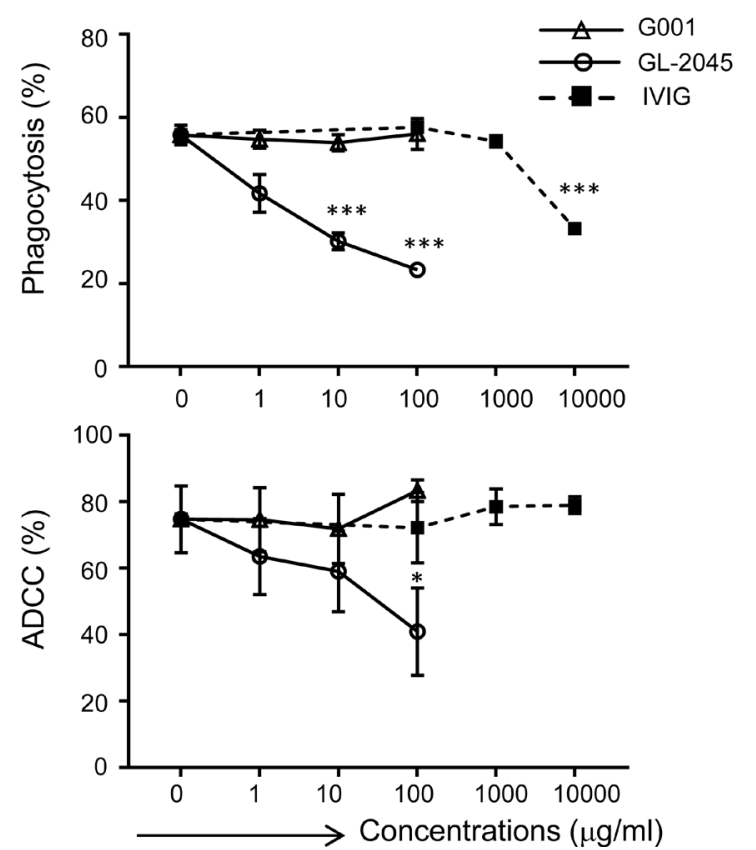
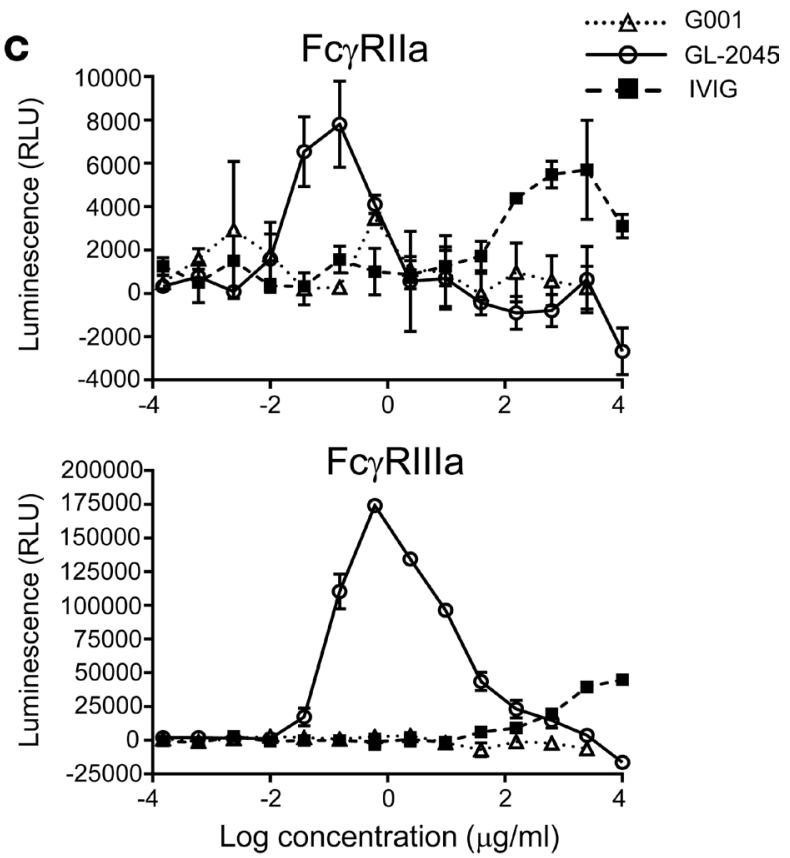

Figure 2. GL-2045 interferes with Fc-Fc $\gamma \mathbf{R}$ interactions and mediates signaling through FcyRIla/Fc $\mathbf{R}$ IIIla. (A) GL-2045 blocks immune complex (IC) - from RA patients $(n=6)$ - binding to human FcyR-expressing CHO cells. Data are shown as mean \pm SEM. (B) GL-2045 interferes with Ab-dependent macrophage phagocytosis (upper) and Ab-dependent NK cytotoxicity (lower). Data are mean \pm SEM of 3 experiments from different donors. (C) GL-2045 mediates signaling through FcyRIla/FcyRIIla. Jurkat cells expressing Fc $\gamma$ RIla or Fc $\gamma$ RIIla were treated with serial dilutions of GL-2045, G001, or IVIC for 6 hours, and nuclear factor of activated T cells (NFAT) pathway activation was measured by luciferase activity quantification. ${ }^{*} P<0.05$, ${ }^{* *} P<0.01,{ }^{* * *} P<0.001$, compared with G001 control (A) or no treatment control (B). One-way ANOVA with Tukey's or Dunnett's multiplicity correction.

paws, as shown by the significantly decreased arthritis scores (Figure 3D). Although M-045, GL-2045, and dexamethasone were associated with numerical decreases in arthritis scores in inflamed paws, these treatment effects did not reach the level of statistical significance (Figure 3E). Histologic analysis of all paws revealed improved outcomes following treatment with dexamethasone, M-045 (data not shown), and GL-2045 (at $50 \mathrm{mg} / \mathrm{kg}$ i.v.) (Figure 4A). When quantified, dexamethasone, M-045 (data not shown), and GL-2045 (at $50 \mathrm{mg} / \mathrm{kg}$ i.v.) mediated statistically significant reductions in inflammation, pannus, cartilage damage, bone resorption, and periosteal bone formation (Figure 4B).

GL-2045 reduces systemic IL-6 levels in CIA. To decipher the mechanisms by which GL-2045 mediated its therapeutic effects in CIA, we analyzed the plasma levels of IL-1 $\beta$, IL-6, IL-10, IFN- $\gamma$, TNF- $\alpha$, and anti-collagen antibodies, all of which have been implicated in the pathophysiology of murine arthritis (35-37). On postenrollment day 5 or 6 , all groups of mice that benefited from GL-2045, M-045, or dexamethasone (as shown by improved arthritis scores) demonstrated significantly lower concentrations of serum IL- 6 compared with PBS-treated CIA mice (Figure 5A). IFN- $\gamma$ and TNF- $\alpha$ levels were not significantly affected 
A
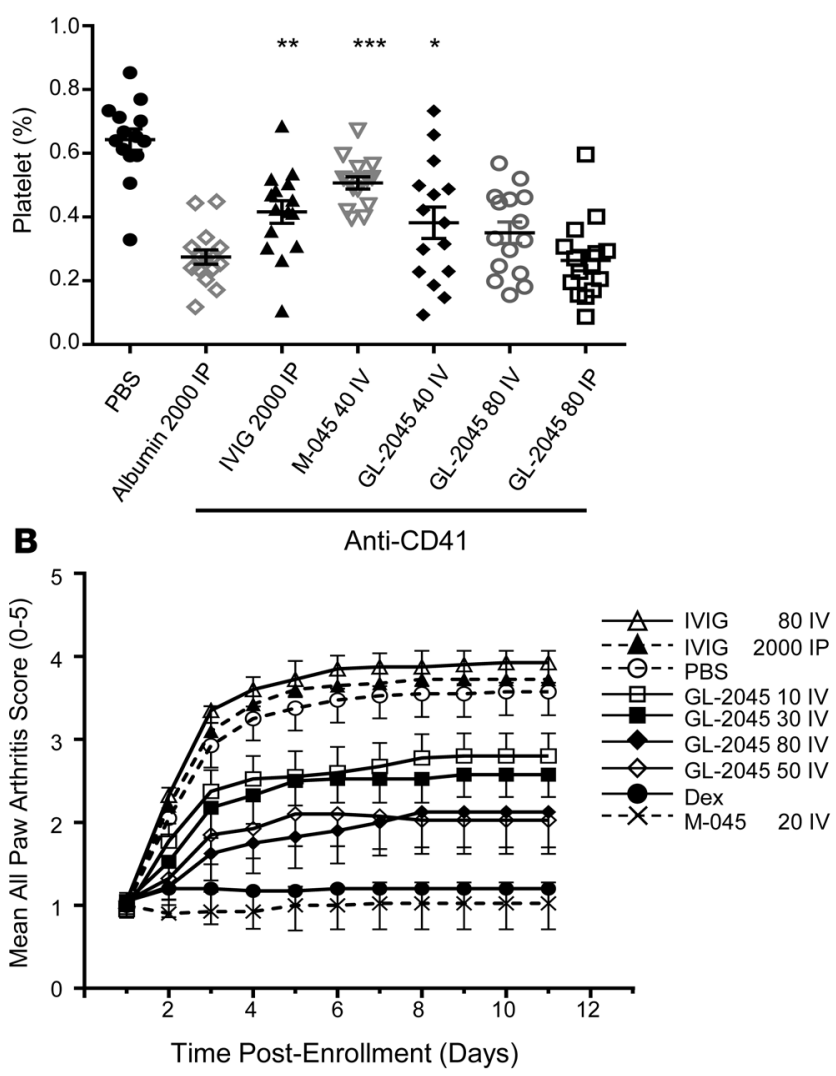

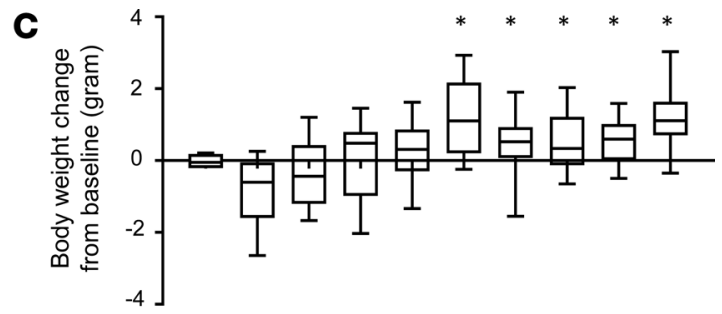

D

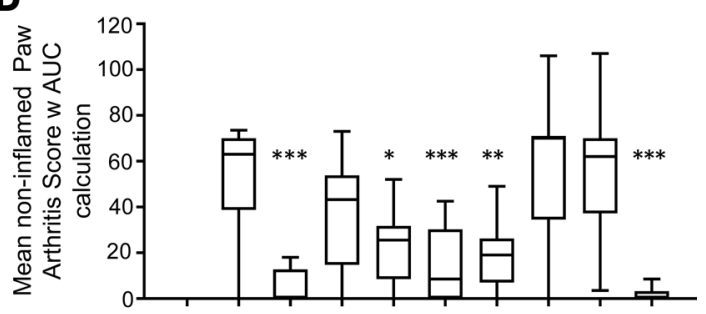

E

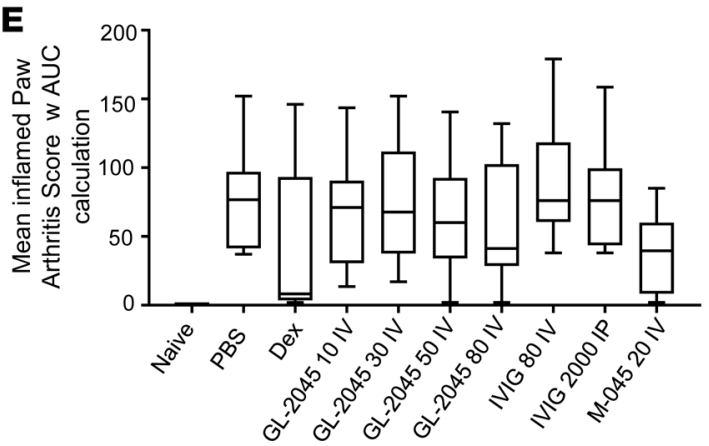

Figure 3. GL-2045 effect in preventing platelet loss in ITP and ameliorating disease activity in CIA. (A) GL-2045 effects on platelet loss in a murine model of ITP. Mice were pretreated with GL-2045 i.v./i.p., M-045 i.v., IVIG i.p., or Albumin i.p. 1 day before platelet depletion with anti-CD41 Ab. The percent of platelets in total cells was analyzed on day 4. Data are shown as dot plot with mean \pm SEM. $n=15$ mice per group. (B) GL-2045 effects on arthritis score in murine CIA. The treatments were initiated after the onset of arthritis, and arthritis scores were evaluated daily after initiation of the treatment. (C) The body weight changes on day 11 after enrollment from baseline were compared among different treatment groups. At the initiation of treatment, individual paws were classified as noninflamed (arthritis score $=0$ ) and inflamed (arthritis score $\geq 1$ ). AUC arthritis scores were calculated and were compared among different treatment groups on noninflamed (D) and inflamed paws (E). (C-E) Data are shown as box and whisker plots (the bounds of the boxes represent the 25th and 75th percentile, the lines represent median, and top and bottom whiskers represent the 95th and 5th percentile). CIA data show naive ( $n=4$ ), diseased $(n=10)$, and treated $(n=10)$. Units for the test compounds: $\mathrm{mg} / \mathrm{kg} .{ }^{*} P<0.05,{ }^{* *} P<0.01,{ }^{* * *} P<0.001$, compared with Albumin group (A) or PBS group (C-E). One-way ANOVA with Dunnett's correction (A, C, D, and E).

by treatment, and IL-1 $\beta$ and IL-10 levels were not detectable (data not shown). Levels of anti-collagen antibodies in GL-2045- and M-045-treated animals showed a trend toward reduction but did not reach statistical significance, compared with PBS-treated mice (Figure 5B). IVIG (2 g/kg i.p.), which failed to effect arthritis scores in this study, demonstrated a statistically significant reduction in anti-collagen antibody levels compared with vehicle-treated controls (Figure 5B).

In order to understand whether these systemic changes reflected those occurring at sites of disease, we employed NanoString technology to measure mRNA expression profiles in the joints of naive, diseased vehicle-treated (PBS), and diseased GL-2045-treated (GL-2045) mice (Supplemental Figure 3 and Supplemental Figure 4). Four samples from the PBS group (all with arthritis scores $>3.6$ ) and 3 samples from the GL-2045 group (with arthritis scores of $0.05,0.7$, and 1.5) did not pass the quality control test; therefore, they were removed from the NanoString analysis (Supplemental Figure 4, A and B). The loss of these subjects may have limited our ability to detect statistically significant changes between cohorts. Diseased PBS-treated mice showed a significant increase in mRNA encoding for IL-1 $\beta$, CCR2, and TGF- $\beta 3$ compared with the naive control mice. Treatment with GL-2045 significantly reduced the levels of CCR2 mRNA compared with the PBS-treated mice. While there was also a reduction in levels of IL-1 $\beta$, IL-6, and TGF- $\beta 3$ between GL-2045-treated and PBS-treated animals, these changes did not reach statistical significance (Supplemental Figure 3C). The importance of IL-1 $\beta$ 
A

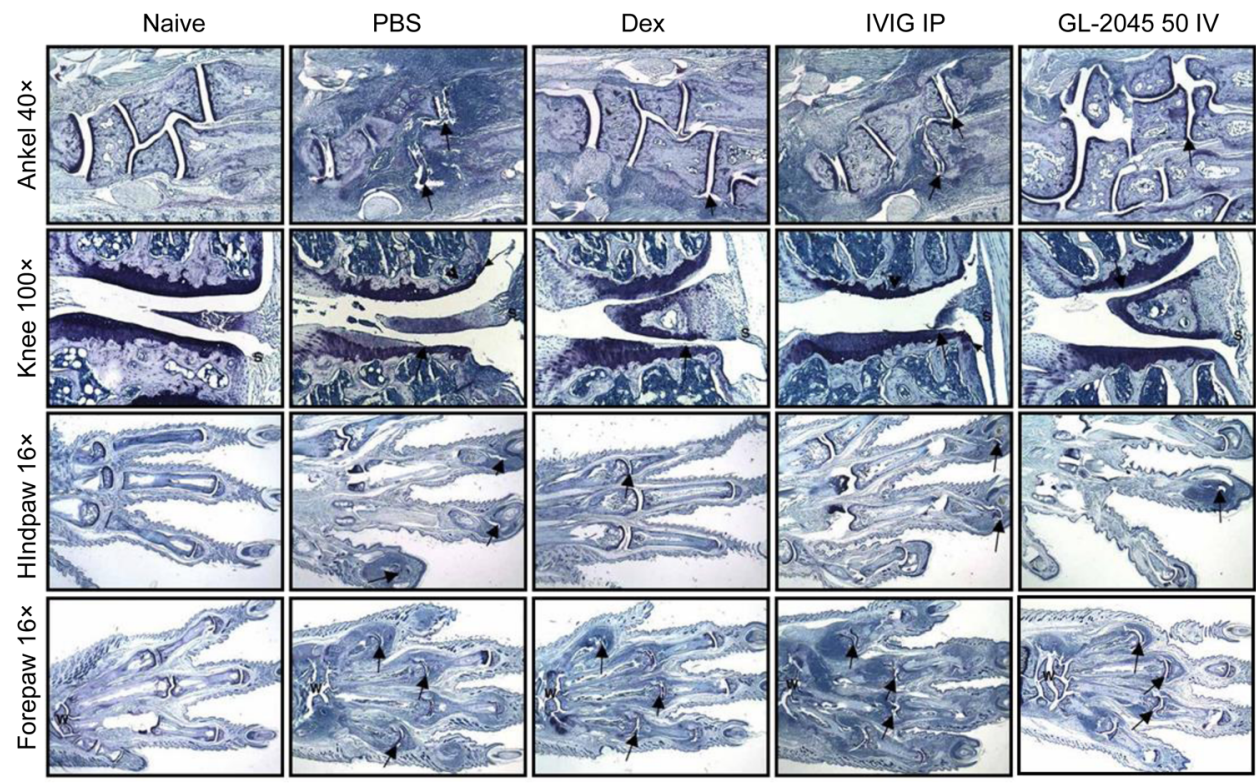

B
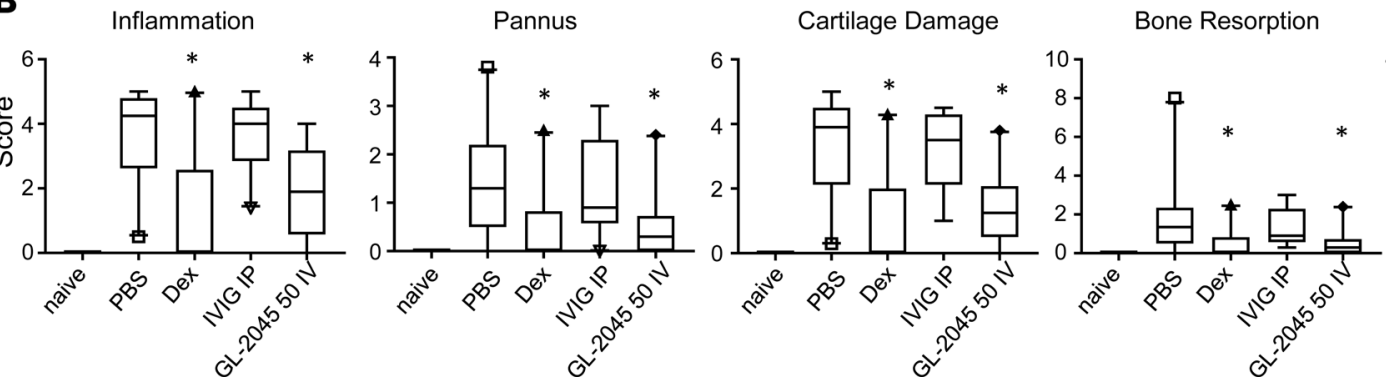

Periosteal Bone Formation

Figure 4. GL-2045 ameliorates histopathological parameters in CIA mice. Joints from each animal were assessed for changes in histopathology parameters at the end of the treatment study (day 11 after enrollment). (A) Representative photomicrographs of toluidine blue-stained ankle (40x magnification), knee (100x magnification), hind paw (16x magnification), and forepaw (16x magnification) joints from selected groups: naive, diseased vehicle-treated (PBS), diseased dexamethasone-treated (Dex), diseased IVIG-treated $2 \mathrm{~g} / \mathrm{kg}$ (IVIG i.p.), and diseased GL-2045-treated $50 \mathrm{mg} / \mathrm{kg}$ (GL-2045 50 i.v.). w, wrist; s, synovium. Arrows point to affected joints. (B) Box and whisker plots of quantification of histopathological parameters measured in all joints from each treatment group. The bounds of the boxes represent the 25 th and 75 th percentile, the lines represent median, and top and bottom whiskers represent the 95th and 5th percentile. Naive mice showed no inflammation or joint pathology. Compared with the PBS group, Dex- and GL-2045-treated animals showed significantly less histopathological evidence of disease. ${ }^{*} P<0.05$ compared with PBS. Kruskal-Wallis test with Dunn's post-hoc test. Naive, $n=4$; all the other groups, $n=10$.

in disease pathogenesis and GL-2045-mediated treatment effects was suggested by its significant association with arthritis score (Supplemental Figure 3D).

$\mathrm{CD} 14^{+}$cells express high levels of $\mathrm{Fc} \gamma \mathrm{Rs}$ and $\mathrm{CD} 14^{+}$inflammatory dendritic cells are known to secrete IL-1 $\beta$, TGF- $\beta$, IL-6, and IL-23 (38). We explored whether CD14 cells were important for GL-2045-mediated inhibition of proinflammatory cytokines and chemokine receptors. Expression analysis revealed that, while CD14 mRNA was reduced in GL-2045-treated vs. PBS-treated diseased mice, it did not reach statistical significance (Supplemental Figure 4A). However, CD14 expression was significantly associated with arthritis score, suggesting the importance of $\mathrm{CD} 14^{+}$cells in disease pathogenesis. Furthermore, the expression of CD14 was significantly correlated with the expression of IL-1 $\beta$ and CCR2, but not IL-6 or TGF- $\beta 3$ (Supplemental Figure 4, B and C).

GL-2045 modulates circulating levels of select cytokines in rats. To understand the effect of GL-2045 on the healthy immune system and as part of the nonclinical development in preparation for future clinical testing, a broad range of doses (1-2000 mg/ $\mathrm{kg}$ ) of GL-2045, administered as i.v. infusions, were evaluated in Wistar Han rats in 2 separate studies (high- and low-dose studies). In the high-dose study, rats received 160,566 , or $2000 \mathrm{mg} / \mathrm{kg}$. However, by day 8 , the $2000 \mathrm{mg} / \mathrm{kg}$ dose had to be lowered to $1200 \mathrm{mg} / \mathrm{kg}$ due to unscheduled euthanasia of 6 rats with clinical observations of limited use and/or swelling of hind limbs; 
A

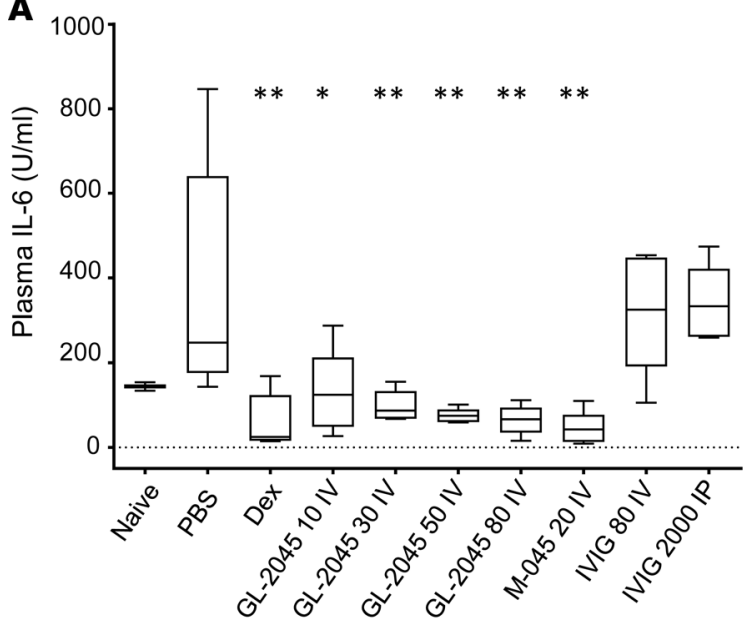

B

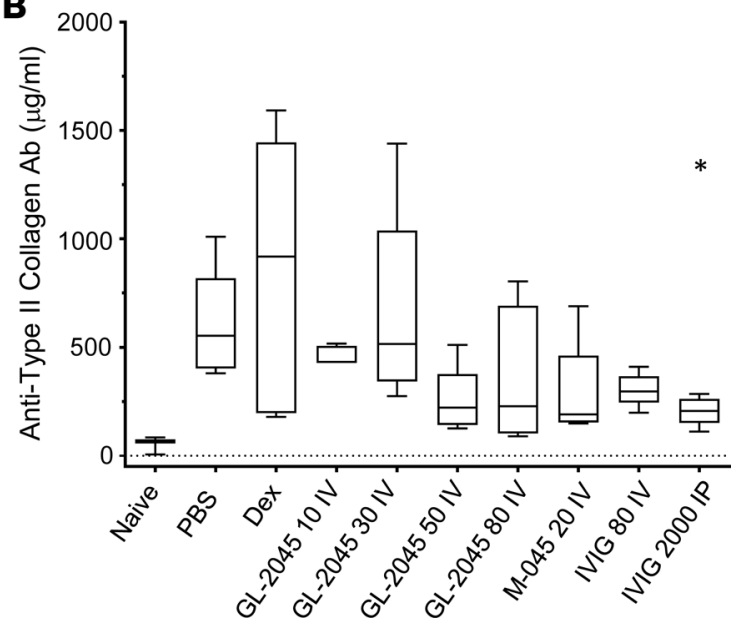

Figure 5. Levels of systemic IL-6 are reduced in GL-2045-treated CIA mice. Blood was collected on day 5 or 6 after enrollment in CIA mice, and plasma levels of IL-6 (A) and anti-type II collagen antibody (B) were measured by ELISA. The bounds of the boxes represent the 25th and 75th percentile, the lines represent median, and top and bottom whiskers represent the 95th and 5th percentile. Naïve, $n=2$; all the other groups, $n=5$. All $P$ values are comparisons against PBS group. ${ }^{*} P<0.05,{ }^{* *} P<0.01$. One-way ANOVA with Dunnett's correction.

therefore, this dose group was labeled 2000/1200 mg/kg. Similar findings were found in some animals receiving lower doses in this study, which also resulted in unscheduled euthanasia. Microscopic evaluations of the hind limbs (data not shown) from euthanized rats provided evidence that indicated the effects were due to complications of the infusion procedure resulting in extravasation of GL-2045 and a local inflammatory response near the infusion site. In the low-dose study in which the $160 \mathrm{mg} / \mathrm{kg}$ dose was also applied, there were only 9 unscheduled euthanasias out of a total of 122 rats (spread across all treatment groups). Although multiple clinical signs were reported, none were deemed to be associated with administration of GL-2045.

Terminal complement complex (TCC; C5b-9) was measured in the low-dose study only. There were no GL-2045-induced changes in TCC at any dose or time point examined (data not shown). Nine cytokines were measured in the serum harvested from the rats in both studies. In general, similar changes in the concentrations of several cytokines were seen within 24 hours after infusion across the 6 weeks of dosing (data not shown); therefore, only the day 1 data are presented (unless indicated). Cytokine concentrations at 0 (preinfusion), 1, 3, and 24 hours after the start of infusion are plotted for IL-10, IL-4, IL-13, and TNF- $\alpha$ (Supplemental Figure 5), and data for the other cytokines measured (IL-2, IL-6, IL-12p70, IL-17, and IFN- $\gamma$ ) are presented in Supplemental Table 1. GL-2045, at the lowest dose tested $(1 \mathrm{mg} / \mathrm{kg})$, had no or limited effects (increased IL-10 at 1 hour on day 1) on the cytokines measured. Small transient changes at 1 and 3 hours after infusion were seen on day 15 for IL-17 and TNF- $\alpha$ and on day 43 for IL-13. At doses $\geq 10 \mathrm{mg} /$ $\mathrm{kg}$, GL-2045 induced statistically significant increases in IL-10, IL-4, IL-13, and TNF- $\alpha$ by 1 or 3 hours after infusion, which either returned to baseline levels (IL-13 and TNF- $\alpha$ ) or trended toward baseline levels (IL10 and IL-4) by 24 hours after infusion. The plots highlight the transient modulation of these cytokines by GL-2045 and, in general, the absence of a dose-response effect as shown by similar concentrations of each cytokine across a broad dose range of GL-2045. The other cytokines measured in this study did not change significantly on day 1 (Supplemental Table 1).

GL-2045 modulates circulating levels of cytokines and complement activation products in NHPs. To understand the effect of GL-2045 on the healthy immune system in a larger animal species and as part of the preclinical development and safety assessment of GL-2045 in preparation for future clinical testing, a broad range of doses (0.1-1000 mg/kg) of GL-2045, administered as i.v. infusions, were evaluated in NHPs. The serum level of GL-2045 increased with increasing dose, with maximum concentration $\left(C_{\max }\right)$ approximately increasing in proportion to dose (Supplemental Figure 6). The overall incidence of anti-drug Ab (ADA) induction to GL-2045 was $62 \%$ (24 of 39 animals) for all GL-2045-dosed animals. The incidence of ADA was $17 \%$ ( 1 of 6 animals), 100\% (6 of 6 animals), 83\% (10 of 12 animals), 83\% (5 of 6 animals), and 22\% (2 of 9 animals) after weekly i.v. administration of GL-2045 at $0.1,10,50,224$, or $1000 \mathrm{mg} / \mathrm{kg} /$ week, respectively. 
The complement activation products $\mathrm{C} 3 \mathrm{a}, \mathrm{C} 4 \mathrm{a}, \mathrm{sC} 5 \mathrm{~b}-9$, and $\mathrm{Bb}$ were transiently increased with no obvious dose related correlations above $10 \mathrm{mg} / \mathrm{kg}$ (data to be reported elsewhere). A panel of eleven cytokines and chemokines were measured in these studies and the effects of GL-2045 on circulating monocyte populations were also monitored. Changes in concentration from baseline (hour 0 for each dose) were evaluated. Similar changes in the levels of cytokines and chemokines measured within 24 hours after infusion were seen across the 6 weeks of dosing (data not shown), so only the day 1 data are presented in which serum concentrations at 0 (preinfusion), 1,3 , and 24 hours are shown.

Our analyses began with a global statistical assessment of the concentration-time profiles of all cytokines and chemokines measured across all doses of GL-2045. The concentration-time profiles of IL-8, IL-1 receptor antagonist (IL-1RA), and IL-10 were significantly different across the doses of GL-2045 ( $P$ $<0.001$ for the low-dose study groups and $P<0.05$ for the high-dose study groups for all 3 markers), and were therefore plotted and considered for further assessment (Figure 6). This included a comparison of the effects of the lowest dose of GL-2045 to the effects in the vehicle group, followed by an assessment of cytokine changes in each GL-2045-dose group compared with the preinfusion baseline levels in that group.

The changes in IL-8, IL-1RA, and IL-10 concentrations induced by GL-2045 dosed at $0.1 \mathrm{mg} / \mathrm{kg}$ (lowest dose) were either no different from or of lower magnitude compared with the vehicle group $(0 \mathrm{mg} / \mathrm{kg})$ group at each time point. Comparing to preinfusion baseline concentrations of these markers, changes in all dose groups were transient, as shown by the recovery to preinfusion concentrations of each marker 24 hours after the start of infusion. In regards to IL-8, a chemokine with proinflammatory activity (39), most of the changes were observed as statistically significant reductions from preinfusion IL- 8 concentrations, in samples collected 1 and 3 hours after infusion. Interestingly, the vehicle group ( $0 \mathrm{mg} / \mathrm{kg}$ ) also showed transiently reduced IL- 8 concentrations that, in general, were not significantly different from those observed in animals receiving GL-2045 at doses $<50 \mathrm{mg} / \mathrm{kg}$ (Figure 6A). However, at doses $\geq 50 \mathrm{mg} / \mathrm{kg}$, these changes from preinfusion baseline levels were greater than those in the vehicle group. At 24 hours, IL- 8 levels were increased above those measured at 3 hours, approaching baseline levels, suggesting a transient modulation of this chemokine by GL-2045 and the vehicle (Figure 6A).

The antiinflammatory cytokines IL-10 and IL-1RA were increased transiently in response to GL-2045, with statistically significant increases in IL-10 seen in the $50 \mathrm{mg} / \mathrm{kg}$ (in the low- but not the high-dose study, 224 , and $1000 \mathrm{mg} / \mathrm{kg}$ groups at 1 hour after infusion and statistically significant increases in IL-1RA detected at 3 hours after infusion at doses $\geq 10 \mathrm{mg} / \mathrm{kg}$ in both studies (Figure 6B). There were no statistically significant changes in IL-2, IL-4, IL-6, IL-12/23p40, IL-13, IL-17, IFN- $\gamma$, or TNF- $\alpha$. However, in the highdose study, there was a trend for reductions in levels of IL-12/23p40, IL-13, and IL-17 in GL-2045-treated animals compared with their individual baselines, while no changes in the vehicle $(0 \mathrm{mg} / \mathrm{kg})$ groups were detected (Supplemental Table 2). There was also a trend for increased IL-6 at several dose levels in the lowdose study (Supplemental Table 2). However, the biologic relevance of these findings was difficult to interpret secondary to a more than 2-fold increase in IL-6 in the vehicle $(0 \mathrm{mg} / \mathrm{kg})$ group in the high-dose study.

GL-2045 induces phenotypic changes on circulating monocytes in NHPs. It is well recognized that cells of monocyte origin are important mediators of the antiinflammatory activities of IVIG (4). GL-2045-induced decreases (compared with baseline, day -9), in peripheral blood monocyte CD14 mean fluorescence intensity (MFI) values, were observed on days 16 and 44 of the dosing phase for nearly all animals only in the highdose study. GL-2045-induced decreases in the percentages of activated monocytes (HLA-DR ${ }^{+}$monocytes) were observed on days 16 and 44 of the dosing phase at doses $\geq 224 \mathrm{mg} / \mathrm{kg}$ (Figure 7). Also, nonsignificant decreases of NK cells were observed in female monkeys only on day 44 at $\geq 224 \mathrm{mg} / \mathrm{kg}$. In the low-dose study, nonsignificant decreases in NK and Tregs were observed in both sexes at $50 \mathrm{mg} / \mathrm{kg}$ (data not shown). Collectively, these data suggest that high doses of GL-2045 and/or associated differences in the infusion paradigms between the low- and high-dose studies differentially influence monocyte expression of CD14 and HLA-DR, with trends for modulation of other immune cell subtypes.

\section{Discussion}

In this study, we report our preclinical evaluation of GL-2045, comprising in vitro analyses of binding and signaling, in vivo studies in rodent models of autoimmunity, and biochemical and cellular changes induced in healthy rats and NHPs. These studies serve as a basis for both the generation of hypotheses to explain the mechanisms by which GL-2045 modulates the immune response and to enable the initiation of clinical testing. 
Low-dose study
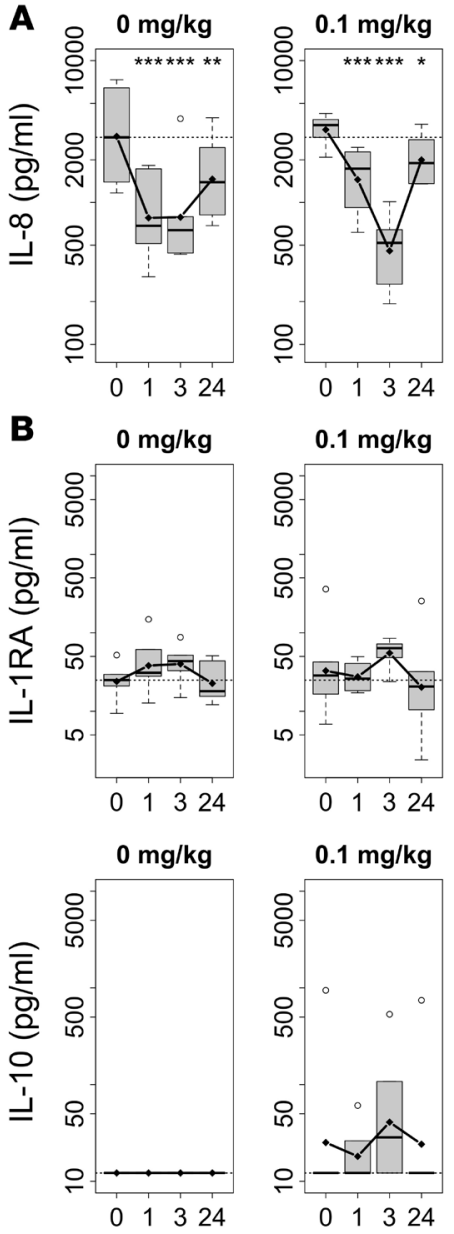
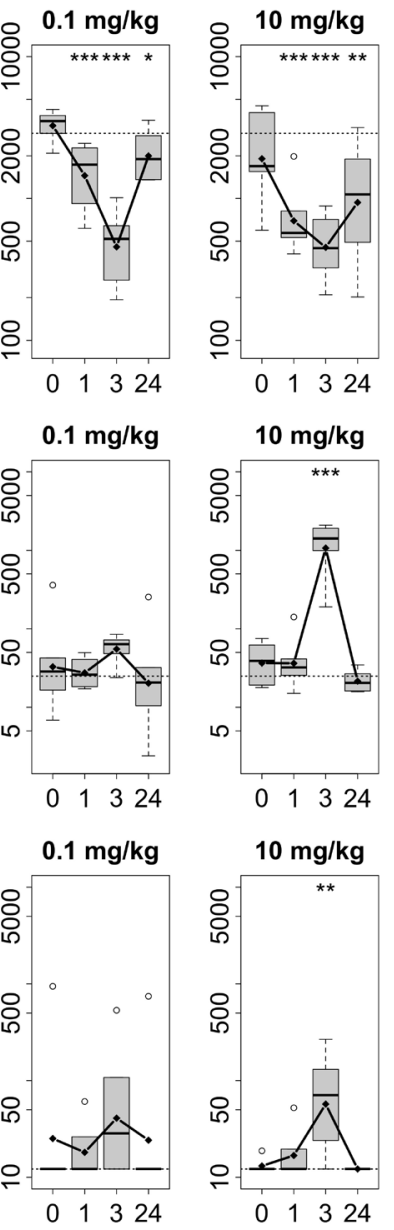
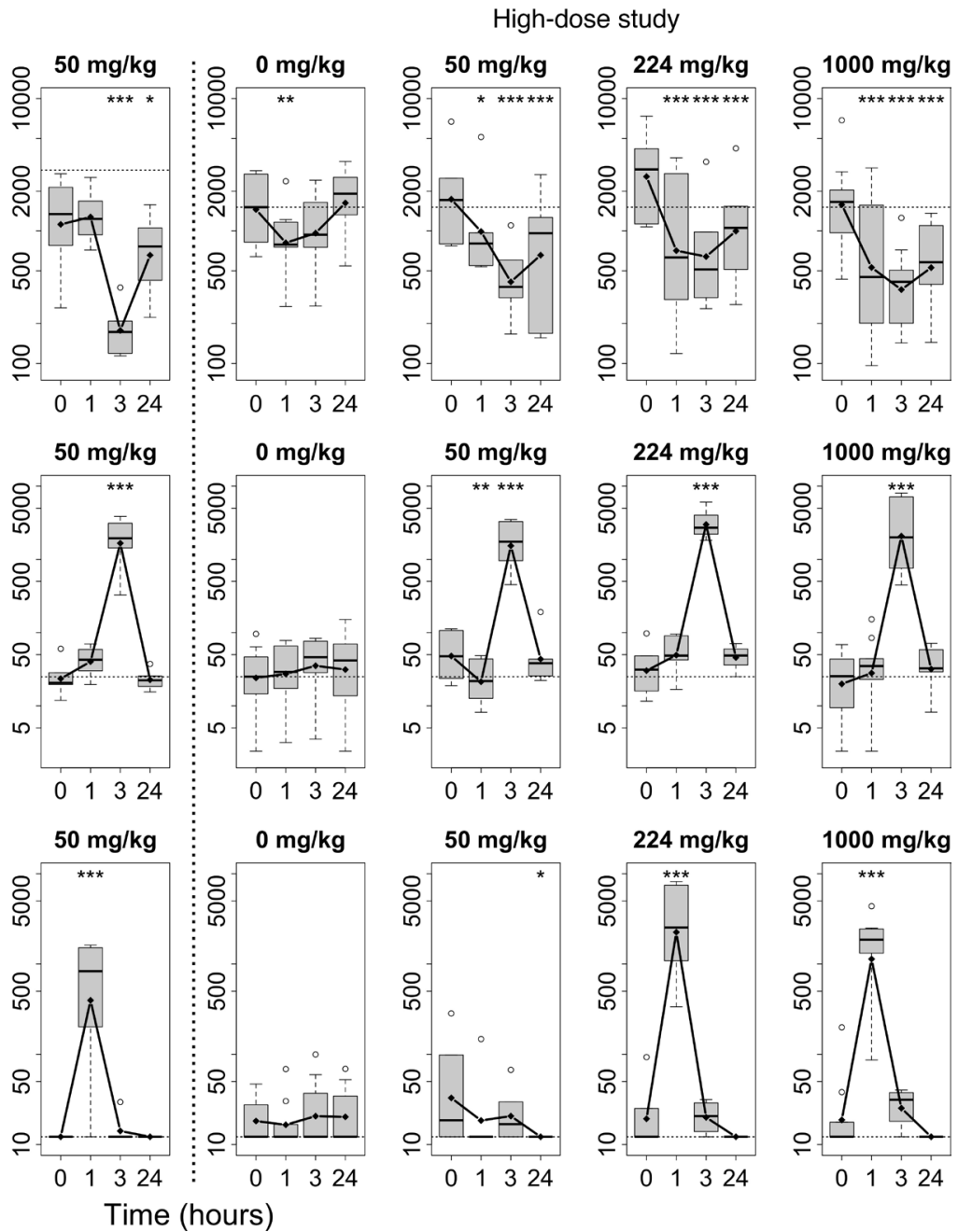

Figure 6. GL-2045 transiently modulates circulating cytokine levels in NHPs. I.v. infusion of CL-2045 decreased IL-8 (A) and increased IL-1RA and IL-10 (B) in the circulation of NHPs. GL-2045 was infused i.v. at different doses in 2 separate studies. Blood samples were collected preinfusion ( 0 hours) and at 1,3 , and 24 hours after initiation of the i.v. infusion, and serum levels of cytokines were assessed. Data from blood collected during the first 24 hours after infusion are shown as least squares means. The bounds of the boxes represent the 25 th and 75 th percentile, the lines represent median, top and bottom whiskers represent the 95th and 5th percentile, and the circles are the values above 95th percentile. Low-dose study: $n=6$ animals per group. High-dose study: $n=9,0 \mathrm{mg} / \mathrm{kg} ; n=$ $6, \mathrm{GL}-2045,50 \mathrm{mg} / \mathrm{kg} ; n=6, \mathrm{GL}-2045,224 \mathrm{mg} / \mathrm{kg} ; n=10, \mathrm{GL}-2045,1000 \mathrm{mg} / \mathrm{kg}$. ${ }^{*} P<0.05,{ }^{* *} P<0.01,{ }^{* * *} P<0.001$, compared with preinfusion levels (0 hours).

Because GL-2045 is composed of highly ordered Fc multimers, which could theoretically vary between lots, we first sought to define the reliability of drug substance production. GL-2045, produced by either Pfizer or Gliknik, yielded a highly consistent protein product as judged by SDS-PAGE gels, size exclusion chromatograms, and binding studies (Supplemental Figure 1). The material generated in the Pfizer laboratories, PF-06755347, was used for the studies in this paper. Based on the knowledge that multimerized Fc fragments are required to engage and signal through the low-affinity Fc $\gamma$ Rs, we initially evaluated the ability of GL-2045 to recapitulate these functions. In vitro studies demonstrated that GL-2045 bound with high avidity to all of the canonical Fc $\gamma$ Rs, inhibited the binding of ICs from patients with RA to Fc $\gamma$ R-expressing $\mathrm{CHO}$ cells, blocked $\mathrm{ADCP}$ and ADCC, and induced signaling through Fc $\gamma \mathrm{RIIa}$ and $\mathrm{F} c \gamma \mathrm{RIII}$. These findings are consistent with recent reports demonstrating that recombinant human IgG1 hexamers and hexamers of IgG4, containing the IgG1 CH3 domain with or without specific point mutations, inhibit select Fc $\gamma$ R engagement and block complement activation (40, 41). Collectively, these data suggest that GL-2045 may be reproducibly generated as a drug product with target-specific functional activity - attributes that made it suitable for characterization in vivo.

GL-2045 is the human analogue of M-045, a recombinant mouse IgG2a Fc multimer that effectively ameliorated autoimmune disease in a variety of rodent models (26). For instance, we previously reported that M-045 was effective in treating CIA and preventing platelet loss in a murine model of ITP. 
Low-dose study
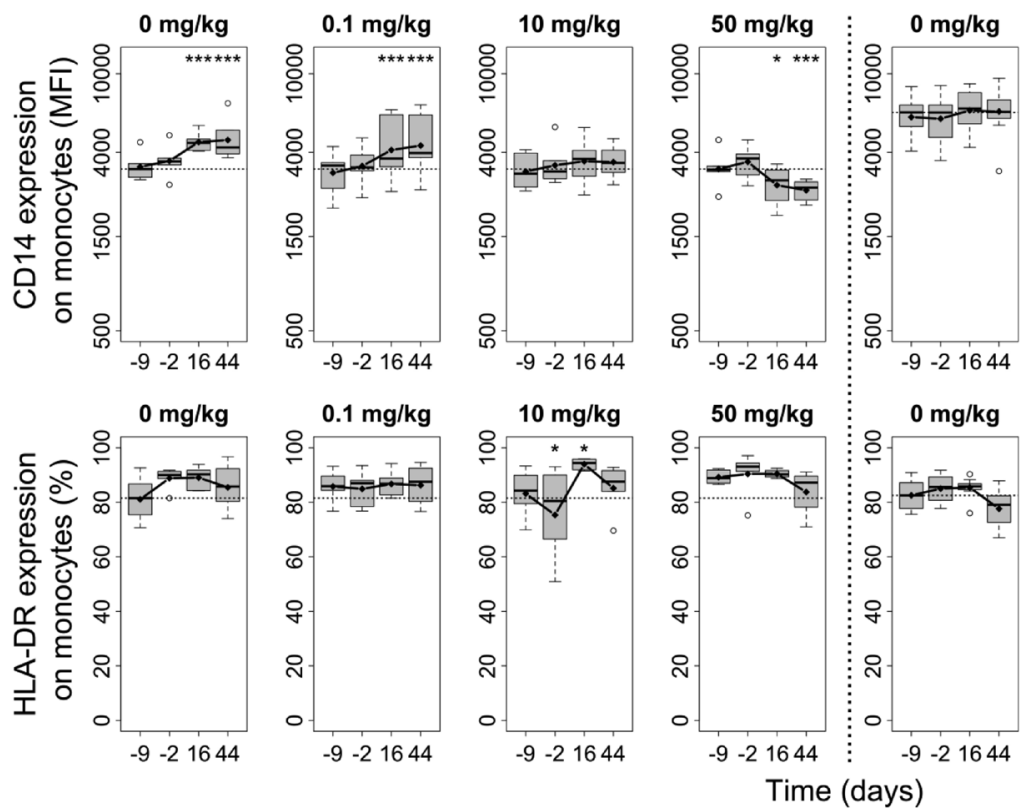

High-dose study
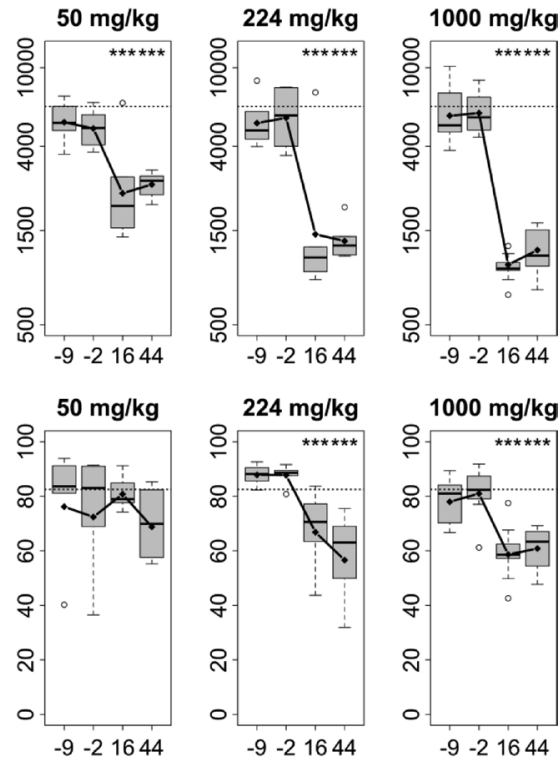

Figure 7. GL-2045 induces phenotypic changes on circulating monocytes in NHPs. GL-2045 was infused i.v. weekly for 6 weeks at different doses. Samples of blood were collected at 9 days ( -9 days) and 2 days ( -2 days) before treatment and 16 and 44 days after initiation of treatment. The expression of CD14 and HLA-DR on monocytes was analyzed by FACS. The bounds of the boxes represent the 25 th and 75 th percentile, the lines represent median, top and bottom whiskers represent the 95 th and 5 th percentile, and the circles are the values above 95 th percentile. ${ }^{*} P<0.05$, ${ }^{* * *} P<0.001$, compared with pretreatment levels (-9 days). Low-dose study: $n=6$ animals per group. High-dose study: $n=10$, vehicle control; $n=6, \mathrm{GL}-2045,50 \mathrm{mg} / \mathrm{kg} ; n=6 \mathrm{GL}-2045,224$ $\mathrm{mg} / \mathrm{kg} ; n=8 \mathrm{GL}-2045,1000 \mathrm{mg} / \mathrm{kg}$.

Additionally, we showed that the ability of M-045 to prevent platelet loss was reduced in Fc $\gamma$ RIIb-deficient mice, with the notable qualification that $\mathrm{Fc}_{\mathrm{RIIb}} \mathrm{R}^{--}$mice had reduced baseline platelet counts compared with WT controls. In order to ascertain whether GL-2045 could recapitulate our observations with M-045 in rodent models of autoimmunity, we evaluated this human drug candidate in similar model systems. GL-2045 improved the arthritis score in a therapeutic CIA model and appeared to protect mice from antibody-mediated platelet loss in the ITP model. M-045 was more efficacious in both models compared with GL-2045, a finding likely due to the fact that M-045 is of mouse origin. These differences are likely not explained by differences in avidity because, based on historical comparisons, M045 and GL-2045 appear to have similar avidity for mouse FcRs (26).

Interestingly, despite the knowledge that autoantibodies play an important role in the pathophysiology of CIA (42), changes in anti-collagen antibody levels were not significantly associated with improved arthritis scores after treatment. This lack of association was likely due to the timing of test article administration, after antibodies were already generated. Additionally, in contrast to recent reports in which IVIG was shown to be effective in preventing CIA and treating both CIA and anti-collagen antibody-induced arthritis $(21,26,43)$, in the current study, IVIG treatment did not demonstrate any therapeutic effects within the 11-day observation period (Figure 3, B-E). We postulate that this discrepancy is attributable to the observation period in the present study, as we have previously reported that IVIG-treated animals have decreased clinical evidence of CIA 2 weeks following initiation of IVIG therapy (26).

The observed changes in systemic and joint-specific cytokine/chemokine levels provide preliminary evidence associating the mechanisms underlying GL-2045 function with suppression of inflammatory cytokines in the treatment of autoimmunity. For example, treatment with GL-2045 significantly reduced systemic levels of IL-6, with a trend toward GL-2045-mediated IL-6 mRNA reduction in arthritic joints. These data are consistent with previous observations showing that IL- 6 is necessary for the development of CIA and that blockade of IL- 6 ameliorates disease $(36,44)$. Additionally, these treatment-related reductions in IL- 6 are likely relevant to the treatment of RA, where the anti-IL- $6 \mathrm{mAb}$, tocilizumab, is approved for the management of patients failing anti-TNF- $\alpha$ therapy (45). 
Furthermore, our findings that GL-2045 reduces CD14 expression in the joint, that CD14 mRNA levels directly correlate with local mRNA levels of IL-1 $\beta$ and CCR2, and that CD14 levels are associated with arthritis score suggest that CD14-expressing monocytes might be of biologic relevance in the antiinflammatory activity of GL-2045. The importance of these monocytes in mediating the antiinflammatory effects of GL-2045 is further supported by our observation that GL-2045 reduced the expression of CD14 and HLA-DR on circulating monocytes in the high-dose NHP study (Figure 7). These changes are potentially biologically relevant because circulating $\mathrm{CD} 14^{+} \mathrm{CD} 16^{+} \mathrm{DR}^{++}$cells, while comprising only $10 \%$ of the monocyte population, are proinflammatory (46). Furthermore, these phenotypic changes mirror those induced by IVIG, where treatment of patients with common variable immunodeficiency results in significant (approximately 50\%) reductions in both $\mathrm{CD} 14^{+} \mathrm{CD} 16^{++}$and $\mathrm{CD} 14^{+} \mathrm{DR}{ }^{+}$monocytes at 4 hours after infusion (47).

We also evaluated the effects of GL-2045 in healthy rats and NHPs to better understand the effect of GL-2045 on the normal/healthy immune system. Our results show that there were differences between rats and NHPs in terms of the cytokines that were modulated by GL-2045. In an effort to elucidate a mechanism for this finding, the binding of GL-2045 to rat immune cells, reported previously (30), was compared with that for NHP immune cells. The pattern of GL-2045 binding to rat immune cells differed from its interactions with immune cells from NHPs, with limited/no binding to NK cells and B cells in rat whole blood and with evidence of binding to the same cell types in NHP whole blood (Figure 1C). This limited binding to rat B cells suggests that GL-2045 may not bind to the inhibitory Fc $\gamma$ RIIb receptor on the surface of circulating immune cells in the rat. Similarly, based on recent studies demonstrating that NK cells are required for the IVIG-mediated prevention of graft vs. host disease (GVHD) in a mouse model, the inability of GL-2045 to engage rat NK cells may also contribute to the different GL-2045-mediated cytokine changes observed in NHPs vs. rats (48).

In NHPs, GL-2045 induced transient increases in IL-1RA and IL-10. A transient suppression of IL-8 levels was seen but only in higher-dose groups ( $\geq 50 \mathrm{mg} / \mathrm{kg}$ ). We postulate that the interstudy differences in cytokine changes in response to GL-2045 dosed at $50 \mathrm{mg} / \mathrm{kg}$ (the only dose that was similar in both studies) are likely secondary to the different infusion paradigms employed in these 2 studies and to interanimal variability. Importantly, GL-2045 did not induce statistically significant increases for any of the well-recognized proinflammatory cytokines measured, including IL-6, IFN- $\gamma$, and TNF- $\alpha$ (Supplemental Table 2), although increases in individual animals were observed for IL-6 at $\geq 10 \mathrm{mg} / \mathrm{kg}$. Interestingly, some of the cytokine changes reported here were similar to those induced by clinically relevant doses (for autoimmune disease) of IVIG in patients with congestive heart failure, in which increased levels of IL-10 and IL-1RA were reported and correlated with improved left ventricular ejection fractions (49).

Collectively, our data provide evidence that treatment with GL-2045 may benefit patients with autoimmune disease. These observations are bolstered by the findings of others, who have recently developed hexameric Fc-fusion proteins or employed our IgG2 hinge multimerization strategy as a basis to develop drug candidates that have functional overlap with GL-2045 $(40,41,50)$. Together with those findings, our studies add to the growing excitement around our concept of using recombinant Fc multimers for the treatment of human autoimmune disease.

\section{Methods}

\section{Preparation of GL-2045}

GL-2045 was produced by Gliknik Inc. or by Pfizer Inc. (PCT application, WO 2017/214321). The drug substance was expressed in $\mathrm{CHO}$ cells and was manufactured using a fed-batch process with chemically defined media. The material was purified using a combination of affinity and ion-exchange chromatography. Analytical characterization ensured that the targeted multimer distribution was achieved. The consistency of GL-2045 between different batches or producers was compared by SDSPAGE, gel filtration, and cell binding. The in vitro blocking of IC binding to CHO-Fc $\gamma \mathrm{R}$ cells and phagocytosis assays were performed with GL-2045 produced by Gliknik Inc. All the in vivo experiments were tested using GL-2045 produced by Pfizer. G001 was prepared by Gliknik Inc. (51). The IVIG used throughout was Gamunex-C (lot no. 13533-800-12/26NNKV1) manufactured by Grifols and human IgG1 (lot no. 11639) was provided by Pfizer.

SDS-PAGE analysis. Nonreduced and reduced SDS-PAGE analyses of GL-2045 were performed as previously described (51). 
Binding studies with immune cells from humans, NHPs, and mice

Heparinized whole blood samples were obtained from healthy human donors (Biological Specialty Corporation) or cynomolgus macaque NHPs. Whole blood $(50 \mu 1)$ was diluted with equal volumes of FACS staining buffer (PBS [Mediatech Inc.], 3\% FBS [Gemini Bio-Products Inc.], 0.05\% NaN3 [MilliporeSigma]) and stained with Dylight 488-labeled GL-2045 (Thermo Fisher Scientific), in combination with the fluorochrome-conjugated anti-human mAbs (CD3 [OKT3, eBioscience], CD14 [HCD14, BioLegend], CD19 [HIB19, BioLegend], and CD56 [B159, BD Biosciences]) or anti-human/NHP mAbs (CD3 [Sp-34-2, BD Biosciences], CD14 [M5E2, BD Biosciences], CD16 [VEP13, Miltenyi Biotec], and CD19 [J3.119, Beckman Coulter]). After 30-minute incubation at room temperature, samples were treated with BD FACS lysis buffer, to lyse RBCs, and were then washed prior to FACS analysis.

A single cell suspension of C57BL/6J mouse spleen was prepared by mincing spleen parenchyma through a cell strainer $(70 \mu \mathrm{m})$, followed by RBC lysis, and washing with PBS. Spleen cells were then incubated with Dylight 488-conjugated GL-2045, in combination with fluorochrome-conjugated anti-mouse mAbs: CD3e (145-2C11, BioLegend), CD45R (RA3-6B2, BD Bioscience), CD49b (DX5, BD Bioscience), CD11b (M1/70, eBioscience), and Gr-1 (RB6-8C5, BioLegend) for 30 minutes at $4^{\circ} \mathrm{C}$. All the samples were acquired using a BD LSRII flow cytometer, and they were analyzed with FACSDiva (BD Bioscience) or Flowjo software (Tree Star Inc.).

\section{Binding studies using BLI}

Binding kinetics were determined by BLI using the Octet Red96 System (software V7.0 or V8.1) (ForteBio). Streptavidin or anti-histidine (anti-His) biosensors were preequilibrated in HBS-EP buffer (catalog BR-100188, GE Healthcare), which is composed of $10 \mathrm{mM}$ HEPES, $150 \mathrm{mM}$ sodium chloride, $3 \mathrm{mM}$ EDTA, $0.005 \%$ polysorbate 20, for a minimum of 10 minutes followed by a 60 -second equilibration in HBS-EP to establish a baseline. Biotinylated or (His) -tagged FcRs (Sino Biologicals) were bound to the biosensors at $0.5 \mu \mathrm{g} / \mathrm{ml}$ for 180 seconds, followed by a 60-second baseline equilibration in HBS-EP. The biosensors were immersed in a solution containing the analytes and were serially diluted from $500 \mu \mathrm{g} / \mathrm{ml}$ for a 600 -second association phase, followed by a 1200-second dissociation phase in HBS-EP. All Fc $\gamma$ R binding was conducted with HBS-EP (pH 7.4).

For FcRn, the anti-(His) ${ }_{6}$ biosensors were preequilibrated in HBS-EP ( $\mathrm{pH}$ 7.4) for a minimum of 10 minutes, followed by a 60-second equilibration in HBS-EP ( $\mathrm{pH} 6.0$ or $\mathrm{pH} 7.4$ ) to establish a baseline. The FcRn were bound to the biosensor at $0.5 \mu \mathrm{g} / \mathrm{ml}$ for 180 seconds ( $\mathrm{pH} 6.0$ or $\mathrm{pH} 7.4$ ), followed by a $60-\mathrm{sec}-$ ond baseline in HBS-EP ( $\mathrm{pH}$ 6.0). The biosensors were immersed in a solution containing the analytes, serially diluted from $500 \mu \mathrm{g} / \mathrm{ml}$ in HBS-EP ( $\mathrm{pH}$ 6.0) for a 600-second association phase, followed by a 1200-second dissociation phase in HBS-EP (pH 7.4). A 1:1 local binding full fit model was used to calculate the apparent binding affinities $\left(\mathrm{K}_{D} \mathrm{~s}\right)$, association $(\mathrm{Ka})$, and dissociation $(\mathrm{Kd})$ rates.

\section{Binding studies using SPR}

DC-SIGN IgG Fc chimera (R\&D Systems) was diluted to $10 \mu \mathrm{g} / \mathrm{ml}$ in Acetate $\mathrm{pH} 5.0$ and amine coupled to a CM5 chip on a Biacore 3000 to an immobilization level of 1600 resonance units (RU). Using HBS-P supplemented with $2 \mathrm{mM}$ calcium chloride as the running and dilution buffers, GL-2045, IVIG, and ICAM-3 were injected at $25 \mu \mathrm{l} /$ minute for 60 seconds. A blank flow cell was used as a reference cell.

\section{IC binding to human Fc $\gamma \mathrm{R}$-expressing $\mathrm{CHO}$ cells (CHO-huFc $\gamma \mathrm{Rs}$ )}

CHO-huFc $\gamma$ Rs were pretreated with G001 or GL-2045 at 10 and $100 \mu \mathrm{g} / \mathrm{ml}$ for 15 minutes at room temperature (51). Serum samples from 6 patients with RA (52), used as source of IC (rheumatoid factor IgG $>60 \mathrm{U} / \mathrm{ml}$ ), were added directly to the culture to achieve a final concentration of $10 \%$ and incubated for 15 minutes at room temperature. Cells were then washed and stained with fluorescein isothiocyanate-labeled goat anti-human IgG (Fab specific) antibody (catalog F5512, MilliporeSigma) and were analyzed by flow cytometry.

FcyRIIa and FcyRIIIa reporter-based signaling assays. Components of the ADCC and ADCP reporter bioassay kits (Promega) were used to measure the effects of GL-2045 on induction of signaling following binding to Fc $\gamma$ RIIIa (V158) and Fc $\gamma$ RIIa (H131), respectively, according to manufacturer's protocol. All compounds were tested in duplicate.

Serially diluted IVIG and GL-2045 were incubated in a volume of $75 \mu$, with the effector cells expressing FcyRIIa or -IIIa for 6 hours at $37^{\circ} \mathrm{C}$. Bio-Glo Luciferase Reagent ( $75 \mu 1 /$ well, Promega) was added and 
incubated for 10 minutes in the dark at room temperature. Results were captured as relative luminescence values using an EnVision 2104 Multi-label reader (Perkin Elmer) and the US LUM 96 program.

\section{Macrophage phagocytosis assay}

In vitro monocyte differentiated macrophages (53) were cocultured with CFSE-labeled (Molecular probe) Ramos cells (ATCC) at a 1:5 ratio in the presence of GL-2045 $(1,10,100 \mu \mathrm{g} / \mathrm{ml})$ for 15 minutes at room temperature in a 96-well ultra-low attachment plate (Corning). Then, anti-CD20 Ab was added directly to the culture at a final concentration of $10 \mu \mathrm{g} / \mathrm{ml}$ for 1 hour at $37^{\circ} \mathrm{C}$. Cells were harvested and stained with Phycoerythrin-conjugated (PE-conjugated) anti-CD11b mAb (catalog 301305, clone ICRF44, BioLegend) for 30 minutes at $4^{\circ} \mathrm{C}$ and analyzed by flow cytometry. Doublets were excluded using forward scatter (FSC) height and width properties. The CFSE/CD11b double-positive cells were identified as phagocytosed macrophages. The percentage of CFSE/CD11b double-positive cells within the total CD11 $\mathrm{b}^{+}$macrophage population was calculated as the capacity of macrophages to phagocytose target cells.

\section{ADCC assay}

PBMCs were separated by density gradient centrifugation from buffy coat (Biological Specialty Inc.), followed by NK cell separation using NK cell isolation kit (Miltenyi Biotec). Increasing concentrations of G001, GL-2045, or IVIG were incubated with NK cells for 5 minutes at room temperature in a 96-well plate, followed by addition of Ramos cells and anti-CD20 Ab at final concentration of $0.1 \mu \mathrm{g} / \mathrm{ml}$. The plate was incubated for 4 hours at $37^{\circ} \mathrm{C}$. The ration of NK/Ramos cells was 3:1. Cells were harvested and stained with CD19 APC (HIB19, BioLegend) and CD56 PE (5.1H11, BioLegend), followed by Annexin V/7-AAD staining in order to quantify B cell death/apoptosis. Doublets were excluded using FSC height and width properties. The Ramos cell gate was first defined on FSC-A vs. side scatter-A (SSC-A) parameters using Ramos cells alone. Then, live Ramos cells were defined as CD19+CD56-/AnnexinV ${ }^{-} / 7-\mathrm{AAD}^{-}$. Cytotoxicity was calculated using the formula: \% cytotoxicity $=(1-$ live Ramos cell number [treated] $/$ [nontreated control]) $\times 100$.

\section{Mouse CIA model}

CIA studies were performed by Bolder BioPATH Inc. Male DBA/1 mice (6-7 weeks old, mean weight $20 \mathrm{~g}$, Harlan Inc.) were administered $150 \mu 1$ of bovine type II collagen (catalog 20021, Chondrex Inc.) in complete Freund's adjuvant (CFA) at the base of the tail on study days 0 and 21. Onset of arthritis occurred between days 18 and 35. Randomization of mice into each test group was done after swelling was established in at least 1 paw (clinical score of 1). Attempts were made to assure approximately equal mean scores of 0.25 /paw across the groups at time of enrollment. Treatments with GL-2045, IVIG, or M-045 were initiated on day 1 following enrollment and dosed Q4D i.v. or i.p. Dexamethasone (VetOne) was orally dosed daily as a positive control treatment, and PBS was used as the vehicle control. Body weights and disease scores for all 4 paws were collected daily after enrollment. Serum was prepared from orbital blood draws on postenrollment days 1 (pretreatment), 2, 5 and 6, and on day 11 via cardiac puncture after euthanasia. Joint tissue was collected for histopathological assessment. Blood samples were analyzed for anti-collagen antibodies using an anti-mouse type II collagen IgG assay kit (Chondrex). Plasma levels of IL-1 $\beta$, IL-6, IL-10, IFN- $\gamma$, and TNF- $\alpha$ were quantified using V-PLEX proinflammatory panel 1 mouse kit (Meso Scale Discovery, MSD K15048D-2).

Formalin-fixed paraffin-embedded (FFPE) joints were sectioned at $8 \mu \mathrm{m}$ and stained with toluidine blue. Six joints from each animal were processed for histopathologic evaluation and scored for inflammation, pannus, cartilage damage, bone resorption, and periosteal new bone formation.

Five $10-\mu \mathrm{m}$ sections were prepared from FFPE samples from each hind paw of individual naive animals, diseased vehicle-treated (PBS), or diseased GL-2045-treated (50 mg/kg i.v. Q4D). Total RNA isolation was performed using an miRNeasy FFPE kit (Qiagen). A panel measuring 561 immunology-related mouse genes was used to measure mRNA counts using nCounter (NanoString Technologies) at Johns Hopkins Deep Sequencing and Microarray Core Facility. The raw gene expression data underwent a series of quality control checks, and flagged samples were excluded from analysis as indicated by nSolver version 2.6 software. A total of 4 samples from untreated animals, 6 from PBS-treated, and 7 from GL-2045-treated samples were suitable for analysis. Data was then normalized using positive, negative, and housekeeping genes (GAPDH, Oaz1, Ppia, Rp119, and Tubb5). The mean \pm 2 times SD of 8 negative 
controls was used for the background subtraction parameters in order to yield a $95 \%$ confidence threshold. The geometric mean of 5 internal positive controls was used to normalize the data across all samples in order to minimize distortion from batch effects.

\section{Mouse ITP model}

Female C57BL/6J mice, age 10-12 weeks, were randomized into study groups of $n=15$. On day 0 , GL-2045 was administered i.v. at 40 and $80 \mathrm{mg} / \mathrm{kg}$ and i.p. at $80 \mathrm{mg} / \mathrm{kg}$. M045 was administered i.v. at $40 \mathrm{mg} / \mathrm{kg}$ and IVIG was dosed i.p. at $2 \mathrm{~g} / \mathrm{kg}$. Additionally, human serum albumin was administered i.p. at $2 \mathrm{~g} / \mathrm{kg}$ as a negative protein load control. A vehicle control was also administered i.p. On days 1-4 of the study, all groups except for the vehicle control group were dosed with anti-CD41 antibody at $68 \mu \mathrm{g} / \mathrm{kg}$ i.p. Blood samples were collected from a lateral tail vein needle stick using a Sarstedt Minivette POCT collection device, on day 0 (prior to the administration of test compounds as baseline) and day 4. Mouse blood samples were stained with anti-CD61-PE (catalog 130-102-628, clone 2C9.G2, Miltenyi Biotec, analyzed with MacsQuant VYB analyzer (Miltenyi Biotec), which determined the percent of platelets in each sample.

\section{In vivo studies in rats}

Two separate rat studies were conducted by Covance Laboratories Inc. (high-dose study) and Charles River Laboratories Montreal ULC (low-dose study), respectively. In both studies, Wistar Han rats were received from Charles River Laboratories. Equal numbers of male and female animals were assigned to different dosing groups using a computerized procedure designed to achieve minimal variation of body weight. Test compounds were diluted with vehicle buffer $(20 \mathrm{mM}$ His, $0.05 \mathrm{mg} / \mathrm{ml}$ EDTA, $5 \%$ sucrose, and $0.2 \mathrm{mg} / \mathrm{ml}$ polysorbate, $\mathrm{pH}$ 5.6) to achieve the desired concentrations. The doses were 1,10 , and $160 \mathrm{mg} / \mathrm{kg}$ (low-dose study) and 160, 566, and 2000/1200 mg/kg (high-dose study). The dose of $2000 \mathrm{mg} / \mathrm{kg}$ was reduced to $1200 \mathrm{mg} / \mathrm{kg}$ beginning on day 8 of the dosing phase due to the unscheduled euthanasia of 6 rats with clinical observations of limited use and/or swelling of hind limbs. Microscopic evaluations of the hind limbs from euthanized rats indicated the effects were due to complications of the infusion procedure resulting in extravasation of GL-2045 and a local inflammatory response near the infusion site. Animals were dosed via weekly i.v. infusions for up to 5 hours/day in the low-dose study and 8 hours/day in the high-dose study (via a catheter implanted in the femoral vein), and blood was drawn on days 1, 15, 29, and 43 at the following intervals: before infusion and 1, 3, and 24 hours after infusion. In the low-dose study, GL-2045 or vehicle was infused at a rate of $0.02 \mathrm{ml} / \mathrm{kg} /$ minute for 1 hour and then at a rate of $0.08 \mathrm{~mL} / \mathrm{kg} / \mathrm{minute}$ for 2.25 hours (for the $10 \mathrm{mg} / \mathrm{kg}$ dose) or 3.91 hours (for the $160 \mathrm{mg} / \mathrm{kg}$ dose). In the high-dose study, the dose rate started at $0.02 \mathrm{ml} / \mathrm{kg} /$ minute and increased to a high-dose rate of $0.09 \mathrm{ml} / \mathrm{kg} / \mathrm{minute}$. Blood samples were collected in serum separator tubes at different time points, and serum samples were assayed for the levels of IFN- $\gamma$, TNF- $\alpha$, IL-2, IL-4, IL-6, IL-10, IL-12p70, IL-13, and IL-17 using the Rat Cytokine/ Chemokine Magnetic Bead Panel (MILLIPLEX Map Assay, MilliporeSigma).

\section{In vivo studies in NHPs}

All of the NHP experiments were performed by Covance Laboratories Inc. Male and female cynomolgus macaques of Mauritius origin were received from Covance Research Products Inc. At the initiation of dosing, animals were 3 years old and weights ranged from $2.8-4.4 \mathrm{~kg}$ for males and 2.6-3.5 kg for females. Animals were randomized using a computerized procedure designed to produce minimal variation of mean body weight for each group/sex. GL-2045 was diluted with the vehicle buffer (20 mM His, $0.05 \mathrm{mg} / \mathrm{ml}$ EDTA, $5 \%$ sucrose, and $0.2 \mathrm{mg} / \mathrm{ml}$ polysorbate, $\mathrm{pH} \mathrm{5.6)}$ and administered via once-weekly i.v. infusions. The doses were $0.1,10$, and $50 \mathrm{mg} / \mathrm{kg}$ (low-dose study) and 50, 224, and $1000 \mathrm{mg} / \mathrm{kg}$ (high-dose study). In the low-dose study, GL-2045 or vehicle was infused at a rate of $0.02 \mathrm{ml} / \mathrm{kg} /$ minute for 1 hour and then at a rate of 0.08 $\mathrm{ml} / \mathrm{kg} /$ minute for 2.25 hours. In the high-dose study, GL-2045 or vehicle was infused at a rate of $0.02 \mathrm{ml} / \mathrm{kg} /$ minute and increased in $0.02 \mathrm{ml} / \mathrm{kg} /$ minute increments approximately every 30 minutes, until a maximum rate of $0.08 \mathrm{ml} / \mathrm{kg} /$ minute was reached. As a precaution against infusion reactions, all animals in the highdose study received Diphenhydramine $\mathrm{HCl}$ via an i.v. bolus injection at $4 \mathrm{mg} / \mathrm{kg}$ approximately 30 minutes prior to the first infusion on each day of dosing. Blood samples were collected from a femoral vein and placed into tubes containing $\mathrm{K}_{2}$ EDTA anticoagulant twice during the prestudy phase and on days 16 (24 hours after the second infusion) and 44 (24 hours after the sixth infusion) of the dosing phase, for immunophenotyping analyses. Blood samples were collected in serum separator tubes at preinfusion and approximately 1,3 , and 
24 hours after the start of first drug infusion for serum cytokine analysis. Serum levels of IL-2, IL-4, IL-6, IL-8, IL-10, IL-13, IL-17, IL-1RA, IL-12p40, IFN- $\gamma$, and TNF- $\alpha$ were quantified using the Milliplex MAP NHP cytokine magnetic bead panel reagent kit (MilliporeSigma, PRCYTOMAG-40K). For immunophenotyping analyses, peripheral blood $(100 \mu \mathrm{l})$ were aliquoted to tubes containing antibody cocktails and incubated for 20 minutes at $4^{\circ} \mathrm{C}$. The following mouse anti-human mAbs were used: CD14 V450 (clone M5E2, BD Biosciences), HLA-DR Alexa Fluor 700 (clone G46-6, BD Biosciences). The RBCs were lysed with $1.5 \mathrm{ml}$ of FACS lysing solution (BD Biosciences). The stained cells were washed 2 times and reconstituted in $300 \mu 1$ of BD staining buffer, analyzed on a BD FACS Canto flow cytometer with DIVA software.

\section{Quantification of GL-2045 in monkey serum}

Blood samples were collected in serum separator tubes on days 1 and 36 of the dosing phase: predose, within 2 minutes, 10 minutes, 30 minutes, and 1, 4, 7, 24, 48, and 96 hours following the end of infusion. An electrochemiluminescent (ECL) immunoassay was validated for the quantification of GL-2045 in monkey serum on the Meso Scale Discovery (MSD) assay platform. Briefly, samples were pretreated with $10 \mathrm{mM}$ dithiothreitol/4\% BSA in PBS with tween $+0.05 \%$ ProClin300 and incubated on a streptavidin-blocked MSD Multi-Array plate coated with biotinylated goat anti-human (total) IgG monkey adsorbed (catalog 2049-08, Southern Biotech). Bound GL-2045 was detected with ruthenium-labeled (Pfizer) mouse anti-human IgG Fc Ab (clone JDC-10, Southern Biotech). The ECL signal was measured using the MSD Sector Imager 6000. Assay responses were quantified in relative light units (RLU). Sample concentrations were determined by interpolation from a standard curve that was fit using a 5-parameter logistic Marquardt regression model with a weighting factor of $1 / \mathrm{y}^{2}$. The range of quantification was $0.0653-4.00 \mu \mathrm{g} / \mathrm{m}$ - in $100 \%$ monkey serum.

\section{Detection of GL-2045 ADA in monkey serum}

Blood samples were collected into serum separator tubes on days 1 and 43 of the dosing phase. An ECL assay was validated to detect the presence of ADA in monkey serum on the MSD assay platform. Briefly, each plate included a positive control, consisting of rabbit anti-GL-2045 polyclonal antibody spiked into monkey serum, and a negative control, consisting of pooled normal monkey serum, to monitor assay performance. Samples were incubated with acid to dissociate potential antibody-drug complexes. Acid dissociated samples were neutralized with Tris-base and then coincubated with biotinylated and ruthenium-labeled GL-2045. Complexes were captured using biotinylated GL-2045 on blocked streptavidin-coated MSD Multi-Array plates. Detection was achieved using tripropylamine to produce an ECL signal that was measured using the MSD instrument. Assay responses were quantified in RLU.

\section{Statistics}

IC binding to Fcy R-expressing CHO cells. To assess inhibition of binding RA ICs at different concentrations of G001 and GL-2045, blood of 6 subjects was exposed to the test compounds at concentrations of 0,10 , and $100 \mu \mathrm{g} / \mathrm{ml}$. One-way ANOVA allowing for unequal variance across treatment groups was used for treatment comparison. All cytokine values were log transformed for analysis. Tukey's correction for multiple comparisons was used to assess significance when all groups were compared with each other. Similarly, 1-way ANOVA with Tukey's multiplicity correction was used for assessment of inhibition of ADCP and ADCC. Dunnett's correction was used when IVIG treatment groups were compared with control.

FCR $\gamma I I a$ and Fcy RIIIa reporter-based signaling assays. Data analysis was performed with GraphPad Prism version 6 software. The background RLUs were subtracted from the RLUs recorded from each well. The $\mathrm{EC}_{50}$ values were determined from the plots of log compound concentration on the $x$ axis vs. RLU values (after background subtraction) on the $y$ axis. A variable slope curve-fitting model (4 parameters) was used within GraphPad Prism 6.0 software. Mean $\mathrm{EC}_{50}$ values \pm SD were calculated from 3 experiments.

ITP study. ITP mouse model was used to assess change in platelet counts in mice $(n=105)$ equally randomized to receive either no intervention or anti-CD41 and 1 of 6 treatments. The assessments were done at baseline and on day 4. One-way ANOVA was used to compare mean platelet percentages across the 7 groups after controlling for baseline.

CIA study. Male DBA/1 mice $(n=104)$ that were $6-7$ weeks old were randomized to 11 groups. Four mice received no intervention, and 100 (10 per group) were randomized to receive vehicle or 1 of 9 treatments after CIA onset was detected. Aggregated over-treatment period arthritis score measure was 
computed by determining AUC. Arthritis scores and biomarker measures were compared using 1-way ANOVA test with multiple comparison adjustment using Dunnett's procedures.

$m R N A$ analysis study. A subset of CIA study mice was used to assess the relationship between arthritis score and mRNA levels of CD14, IL-1 $\beta$, IL-6, CCR2, and TGF- $\beta 3$. All naive $(n=4)$, diseased vehicle-treated $(n=6)$, and diseased GL-2045-treated $50 \mathrm{mg} / \mathrm{kg}(n=7)$ mice were analyzed. The groups were compared using 1-way ANOVA with Tukey correction. Linear models adjusted for treatment group were used to test significance of the linear associations of the measures.

In vivo studies in rats and NHPS. Two studies were performed in Wistar Han rats and 2 in cynomolgus macaque monkeys. In the rat studies, male and female Wistar Han rats were equally randomized to receive different doses of GL-2045. In the low-dose study, $0 \mathrm{mg} / \mathrm{kg}$ (20 animals), $1 \mathrm{mg} / \mathrm{kg}$ (20 animals), $10 \mathrm{mg} /$ $\mathrm{kg}$ (20 animals), and $160 \mathrm{mg} / \mathrm{kg}$ (20 animals) were the doses given. In the high-dose study, $0 \mathrm{mg} / \mathrm{kg}$ (30 animals), $160 \mathrm{mg} / \mathrm{kg}$ (20 animals), $566 \mathrm{mg} / \mathrm{kg}$ (20 animals), and 2000/1200 mg/kg (30 animals) were the doses given. Blood was drawn on days 1, 15, 29, and 43 at the following intervals: preinfusion and 1, 3, and 24 hours after infusion. For this document, only analyses of the day-1 samples are reported.

In the NHP studies, male and female NHPs were equally randomized to receive different doses of GL-2045 as follows: low-dose study, $0 \mathrm{mg} / \mathrm{kg}$ ( 6 animals), $0.1 \mathrm{mg} / \mathrm{kg}$ (6 animals), $10 \mathrm{mg} / \mathrm{kg}$ (6 animals), and $50 \mathrm{mg} / \mathrm{kg}$ (6 animals); high-dose study, $0 \mathrm{mg} / \mathrm{kg}$ (10 animals), $50 \mathrm{mg} / \mathrm{kg}$ (6 animals), $224 \mathrm{mg} / \mathrm{kg}$ (6 animals), and $1000 \mathrm{mg} / \mathrm{kg}$ (10 animals). Blood was drawn for assessments on days 1, 15, 29, and 43 at the following intervals: preinfusion, 1, 3, and 24 hours after infusion. For this document, only analyses of the day-1 samples are reported.

Analysis of rat and NHP cytokines/chemokines. All cytokine/chemokine values were log transformed for analysis. A mixed effect model was used to assess repeated measures, accounting for correlation of observations within each animal. Each model included treatment, time, sex, and treatment by time-interaction term. The compound symmetry covariance structure was specified. The significance of interaction term was used to test the treatment group effect. Bonferroni correction was used to adjust for multiple comparisons. Least squares means and corresponding 95\% CI were reported for each treatment group at each time point. Data distributions were assessed for the normality assumption with stem and leaf, box plots, Q-Q plots, and the Shapiro-Wilk statistic. All analyses were produced using GraphPad PRISM, R 3.3.2 , and SAS version 9.4 (SAS Institute Inc.).

\section{Study Approval}

Blood samples from patients with RA were acquired from anonymized patients at the Mayo Clinic. The protocol was approved by the IRB at the Mayo Clinic College of Medicine and the University of Maryland School of Medicine, and all patients provided informed written consent. The mouse CIA model experiments were conducted by Bolder BioPATH Inc. The study design and animal usage was approved by Bolder BioPATH's IACUC. The mouse ITP model studies were performed by Pfizer. All procedures were done in accordance with the Pfizer IACUC and the NIH Guide for the Care and Use of Laboratory Animals. The NHP studies and high-dose rat study were performed in Covance Laboratories. The low dose rat study was conducted by Charles River Laboratories Montreal ULC. All procedures in the protocol were in compliance with applicable animal welfare acts and were approved by the local IACUC.

\section{Author contributions}

$\mathrm{XZ}$, JO, and HSO contributed equally to this paper. GL and SES share senior authorship. XZ, JO, HSO, DSB, GL, and SES all contributed to the conceptual design of the study and interpretation of the results. EYM and HSO produced and characterized the proteins for the in vitro studies. DB and DR provided statistical oversight for all studies and defined the appropriate statistical methodologies. XZ performed in vitro $\mathrm{CHO}$ cell binding, $\mathrm{ADCC}$, and $\mathrm{ADCP}$ assays. $\mathrm{XZ}$ and $\mathrm{HZ}$ performed immune cell binding assays. $\mathrm{EB}$ performed the Biacore studies and analyzed the data. XL and MCM performed the binding and luciferase assays, and MCM performed the BLI assays. GLW performed PK and ADA assays. ES and HH were responsible for the NanoString studies and associated data analysis.

\section{Acknowledgments}

Experiments conducted at the University of Maryland SOM were funded by Gliknik through a sponsored Research Agreement with the University of Maryland Baltimore. We thank Jessica Whritenour, Declan Flynn, Phil Bartholomew, and Lynn O'Donnell (Pfizer) for their careful review of this manuscript. 
Address correspondence to: Scott E. Strome, University of Tennessee Health Science Center College of Medicine, 910 Madison Avenue, 10th floor, Suite 1002, Memphis, Tennessee 38163, USA. Phone: 901.448.5293; Email: sstrome@uthsc.edu.

SES's present address is: University of Tennessee Health Science Center College of Medicine, Memphis, Tennessee, USA.

1. Gelfand EW. Intravenous immune globulin in autoimmune and inflammatory diseases. N Engl J Med. 2012;367(21):2015-2025.

2. Lünemann JD, Nimmerjahn F, Dalakas MC. Intravenous immunoglobulin in neurology--mode of action and clinical efficacy. Nat Rev Neurol. 2015;11(2):80-89.

3. Schwab I, Nimmerjahn F. Intravenous immunoglobulin therapy: how does IgG modulate the immune system? Nat Rev Immunol. 2013;13(3):176-189.

4. Nimmerjahn F, Ravetch JV. The antiinflammatory activity of IgG: the intravenous IgG paradox. J Exp Med. 2007;204(1):11-15.

5. Clynes R. Protective mechanisms of IVIG. Curr Opin Immunol. 2007;19(6):646-651.

6. Anthony RM, Kobayashi T, Wermeling F, Ravetch JV. Intravenous gammaglobulin suppresses inflammation through a novel $\mathrm{T}(\mathrm{H}) 2$ pathway. Nature. 2011;475(7354):110-113.

7. Anthony RM, Nimmerjahn F, Ashline DJ, Reinhold VN, Paulson JC, Ravetch JV. Recapitulation of IVIG anti-inflammatory activity with a recombinant IgG Fc. Science. 2008;320(5874):373-376.

8. Anthony RM, Wermeling F, Karlsson MC, Ravetch JV. Identification of a receptor required for the anti-inflammatory activity of IVIG. Proc Natl Acad Sci USA. 2008;105(50):19571-19578.

9. Kaneko Y, Nimmerjahn F, Ravetch JV. Anti-inflammatory activity of immunoglobulin G resulting from Fc sialylation. Science. 2006;313(5787):670-673.

10. Su Y, Rossi R, De Groot AS, Scott DW. Regulatory T cell epitopes (Tregitopes) in IgG induce tolerance in vivo and lack immunogenicity per se. J Leukoc Biol. 2013;94(2):377-383.

11. Siragam V, Brinc D, Crow AR, Song S, Freedman J, Lazarus AH. Can antibodies with specificity for soluble antigens mimic the therapeutic effects of intravenous IgG in the treatment of autoimmune disease? J Clin Invest. 2005;115(1):155-160.

12. Siragam V, Crow AR, Brinc D, Song S, Freedman J, Lazarus AH. Intravenous immunoglobulin ameliorates ITP via activating Fc gamma receptors on dendritic cells. Nat Med. 2006;12(6):688-692.

13. Lutz HU, et al. Intravenously applied IgG stimulates complement attenuation in a complement-dependent autoimmune disease at the amplifying C3 convertase level. Blood. 2004;103(2):465-472.

14. Li N, et al. Complete FCRn dependence for intravenous Ig therapy in autoimmune skin blistering diseases. J Clin Invest. 2005;115(12):3440-3450.

15. Janczy JR, et al. Immune complexes inhibit IL-1 secretion and inflammasome activation. J Immunol. 2014;193(10):5190-5198

16. Leontyev D, Katsman Y, Ma XZ, Miescher S, Käsermann F, Branch DR. Sialylation-independent mechanism involved in the amelioration of murine immune thrombocytopenia using intravenous gammaglobulin. Transfusion. 2012;52(8):1799-1805

17. Leontyev D, Katsman Y, Branch DR. Mouse background and IVIG dosage are critical in establishing the role of inhibitory Fcy receptor for the amelioration of experimental ITP. Blood. 2012;119(22):5261-5264.

18. Levin D, Golding B, Strome SE, Sauna ZE. Fc fusion as a platform technology: potential for modulating immunogenicity. Trends Biotechnol. 2015;33(1):27-34.

19. Orange JS, et al. Use of intravenous immunoglobulin in human disease: a review of evidence by members of the Primary Immunodeficiency Committee of the American Academy of Allergy, Asthma and Immunology. J Allergy Clin Immunol. 2006;117(4 Suppl):S525-S553.

20. Späth PJ, Granata G, La Marra F, Kuijpers TW, Quinti I. On the dark side of therapies with immunoglobulin concentrates: the adverse events. Front Immunol. 2015;6:11.

21. Campbell IK, et al. Therapeutic effect of IVIG on inflammatory arthritis in mice is dependent on the Fc portion and independent of sialylation or basophils. J Immunol. 2014;192(11):5031-5038.

22. Yu X, Vasiljevic S, Mitchell DA, Crispin M, Scanlan CN. Dissecting the molecular mechanism of IVIg therapy: the interaction between serum IgG and DC-SIGN is independent of antibody glycoform or Fc domain. J Mol Biol. 2013;425(8):1253-1258.

23. Sharma M, et al. Intravenous immunoglobulin-induced IL-33 is insufficient to mediate basophil expansion in autoimmune patients. Sci Rep. 2014;4:5672.

24. von Gunten S, et al. IVIG pluripotency and the concept of Fc-sialylation: challenges to the scientist. Nat Rev Immunol. 2014;14(5):349.

25. Nagelkerke SQ, et al. Inhibition of Fc $\gamma R$-mediated phagocytosis by IVIg is independent of IgG-Fc sialylation and Fc $\gamma$ RIIb in human macrophages. Blood. 2014;124(25):3709-3718.

26. Jain A, et al. Fully recombinant IgG2a Fc multimers (stradomers) effectively treat collagen-induced arthritis and prevent idiopathic thrombocytopenic purpura in mice. Arthritis Res Ther. 2012;14(4):R192.

27. Niknami M, Wang MX, Nguyen T, Pollard JD. Beneficial effect of a multimerized immunoglobulin Fc in an animal model of inflammatory neuropathy (experimental autoimmune neuritis). J Peripher Nerv Syst. 2013;18(2):141-152.

28. Thiruppathi M, Sheng JR, Li L, Prabhakar BS, Meriggioli MN. Recombinant IgG2a Fc (M045) multimers effectively suppress experimental autoimmune myasthenia gravis. J Autoimmun. 2014;52:64-73.

29. Zhou H, et al. A fully recombinant human IgG1 Fc multimer (GL-2045) inhibits complement-mediated cytotoxicity and induces iC3b. Blood Adv. 2017;1(8):504-515.

30. Sun $\mathrm{H}$, et al. Recombinant human IgG1 based Fc multimers, with limited FcR binding capacity, can effectively inhibit complement-mediated disease. J Autoimmun. 2017;84:97-108.

31. Roopenian DC, Akilesh S. FcRn: the neonatal Fc receptor comes of age. Nat Rev Immunol. 2007;7(9):715-725. 
32. Wilson TJ, Fuchs A, Colonna M. Cutting edge: human FcRL4 and FcRL5 are receptors for IgA and IgG. J Immunol. 2012;188(10):4741-4745.

33. Pincetic A, et al. Type I and type II Fc receptors regulate innate and adaptive immunity. Nat Immunol. 2014;15(8):707-716.

34. Boekhoudt GH, Frazier-Jessen MR, Feldman GM. Immune complexes suppress IFN-gamma signaling by activation of the FcgammaRI pathway. J Leukoc Biol. 2007;81(4):1086-1092.

35. Fujimoto M, et al. Interleukin-6 blockade suppresses autoimmune arthritis in mice by the inhibition of inflammatory Th17 responses. Arthritis Rheum. 2008;58(12):3710-3719.

36. Alonzi T, et al. Interleukin 6 is required for the development of collagen-induced arthritis. J Exp Med. 1998;187(4):461-468.

37. Joosten LA, Helsen MM, van de Loo FA, van den Berg WB. Anticytokine treatment of established type II collagen-induced arthritis in DBA/1 mice: a comparative study using anti-TNFalpha, anti-IL-1alpha/beta and IL-1Ra. Arthritis Rheum. 2008;58(2 Suppl):S110-S122.

38. Segura E, et al. Human inflammatory dendritic cells induce Th17 cell differentiation. Immunity. 2013;38(2):336-348.

39. Russo RC, Garcia CC, Teixeira MM, Amaral FA. The CXCL8/IL-8 chemokine family and its receptors in inflammatory diseases. Expert Rev Clin Immunol. 2014;10(5):593-619.

40. Qureshi OS, et al. Multivalent Fcy-receptor engagement by a hexameric Fc-fusion protein triggers Fcy-receptor internalisation and modulation of Fc $\gamma$-receptor functions. Sci Rep. 2017;7(1):17049.

41. Tradtrantip L, Felix CM, Spirig R, Morelli AB, Verkman AS. Recombinant IgG1 Fc hexamers block cytotoxicity and pathological changes in experimental in vitro and rat models of neuromyelitis optica. Neuropharmacology. 2018;133:345-353.

42. Batsalova T, Lindh I, Bäcklund J, Dzhambazov B, Holmdahl R. Comparative analysis of collagen type II-specific immune responses during development of collagen-induced arthritis in two B10 mouse strains. Arthritis Res Ther. 2012;14(6):R237.

43. Svetlicky N, et al. Anti-citrullinated-protein-antibody-specific intravenous immunoglobulin attenuates collagen-induced arthritis in mice. Clin Exp Immunol. 2015;182(3):241-250.

44. Takagi N, et al. Blockage of interleukin-6 receptor ameliorates joint disease in murine collagen-induced arthritis. Arthritis Rheum. 1998;41(12):2117-2121.

45. Emery P, et al. IL-6 receptor inhibition with tocilizumab improves treatment outcomes in patients with rheumatoid arthritis refractory to anti-tumour necrosis factor biologicals: results from a 24-week multicentre randomised placebo-controlled trial. Ann Rheum Dis. 2008;67(11):1516-1523.

46. Belge KU, et al. The proinflammatory CD14+CD16+DR++ monocytes are a major source of TNF. J Immunol. 2002;168(7):3536-3542

47. Siedlar M, et al. Preparations of intravenous immunoglobulins diminish the number and proinflammatory response of CD14+CD16++ monocytes in common variable immunodeficiency (CVID) patients. Clin Immunol. 2011;139(2):122-132

48. Gregoire-Gauthier J, et al. Role of Natural Killer Cells in Intravenous Immunoglobulin-Induced Graft-versus-Host Disease Inhibition in NOD/LtSz-scidIL2rg(-/-) (NSG) Mice. Biol Blood Marrow Transplant. 2015;21(5):821-828.

49. Gullestad L, et al. Immunomodulating therapy with intravenous immunoglobulin in patients with chronic heart failure. Circulation. 2001;103(2):220-225

50. Ortiz DF, et al. Elucidating the interplay between IgG-Fc valency and Fc $\gamma$ R activation for the design of immune complex inhibitors. Sci Transl Med. 2016;8(365):365ra158.

51. Zhang X, et al. Anti-CD20 Antibody with Multimerized Fc Domains: A Novel Strategy To Deplete B Cells and Augment Treatment of Autoimmune Disease. J Immunol. 2016;196(3):1165-1176.

52. Zhang X, et al. CD40 mediates downregulation of CD32B on specific memory B cell populations in rheumatoid arthritis. J Immunol. 2013;190(12):6015-6022

53. Chen S, So EC, Strome SE, Zhang X. Impact of Detachment Methods on M2 Macrophage Phenotype and Function. J Immunol Methods. 2015;426:56-61. 